ARTICLE

\title{
Targeting miR-126 in inv(16) acute myeloid leukemia inhibits leukemia development and leukemia stem cell maintenance
}

Lianjun Zhang ${ }^{1,8}$, Le Xuan Truong Nguyen ${ }^{1,8}$, Ying-Chieh Chen ${ }^{1}$, Dijiong Wu (i) ${ }^{2}$, Guerry J. Cook , Dinh Hoa Hoang ${ }^{1}$, Casey J. Brewer ${ }^{1}$, Xin He${ }^{1}$, Haojie Dong ${ }^{1}$, Shu $\mathrm{Li}^{3}$, Man Li ${ }^{1}$, Dandan Zhao ${ }^{1}$, Jing Qi ${ }^{1}$, Wei-Kai Hua ${ }^{1}$, Qi Cai ${ }^{1}$, Emily Carnahan', Wei Chen ${ }^{4}$, Xiwei Wu${ }^{4}$, Piotr Swiderski ${ }^{5}$, Russell C. Rockne (1) ${ }^{6}$, Marcin Kortylewski ${ }^{7}{ }^{7}$, Ling $\mathrm{Li}^{1}$, Bin Zhang (i] ${ }^{1}$, Guido Marcucci ${ }^{1}{ }^{1} \&$ Ya-Huei Kuo ${ }^{1 凶}$

Acute myeloid leukemia ( $A M L)$ harboring inv(16)(p13q22) expresses high levels of miR-126. Here we show that the CBFB-MYH11 (CM) fusion gene upregulates miR-126 expression through aberrant miR-126 transcription and perturbed miR-126 biogenesis via the HDAC8/ RAN-XPO5-RCC1 axis. Aberrant miR-126 upregulation promotes survival of leukemiainitiating progenitors and is critical for initiating and maintaining CM-driven AML. We show that miR-126 enhances MYC activity through the SPRED1/PLK2-ERK-MYC axis. Notably, genetic deletion of miR-126 significantly reduces $A M L$ rate and extends survival in CM knockin mice. Therapeutic depletion of miR-126 with an anti-miR-126 (miRisten) inhibits AML cell survival, reduces leukemia burden and leukemia stem cell (LSC) activity in inv(16) AML murine and xenograft models. The combination of miRisten with chemotherapy further enhances the anti-leukemia and anti-LSC activity. Overall, this study provides molecular insights for the mechanism and impact of miR-126 dysregulation in leukemogenesis and highlights the potential of miR-126 depletion as a therapeutic approach for inv(16) AML.

\footnotetext{
${ }^{1}$ Gehr Family Center for Leukemia Research, Department of Hematological Malignancies Translational Science, Hematologic Malignancies and Stem Cell Transplantation Institute, Beckman Research Institute, City of Hope Medical Center, Duarte, CA 91010, USA. ${ }^{2}$ Department of Hematology, First Affiliated Hospital of Zhejiang Chinese Medical University, Hangzhou, Zhejiang 310006, China. ${ }^{3}$ Department of Hematology, The Second Affiliated Hospital, Zhejiang University School of Medicine, Hangzhou, Zhejiang 310009, China. ${ }^{4}$ Integrated Genomics Core, Beckman Research Institute, City of Hope Medical Center, Duarte, CA 91010, USA. ${ }^{5}$ Department of Molecular Medicine, Beckman Research Institute, City of Hope Medical Center, Duarte, CA 91010, USA.

${ }^{6}$ Department of Computational and Quantitative Medicine, Division of Mathematical Oncology, Beckman Research Institute, City of Hope Medical Center, Duarte, CA 91010, USA. ${ }^{7}$ Department of Immuno-oncology, Beckman Research Institute, City of Hope Medical Center, Duarte, CA 91010, USA. ${ }^{8}$ These

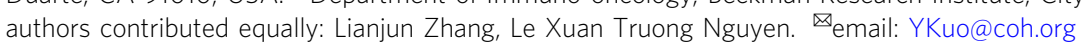


A cute myeloid leukemia (AML) is an aggressive hematopoietic malignancy characterized by excessive proliferation of immature leukemic blasts. Approximately 21,000 patients are diagnosed with AML each year in the United States, and the latest 5 -year overall survival rate remains at only $28 \%$ (https://seer.cancer.gov) ${ }^{1,2}$. AML is maintained and propagated by a population of leukemia-initiating cells or leukemia stem cells (LSCs), which feature quiescence and therapy resistance, and contribute to subsequent clonal evolution of the disease and relapse $^{3,4}$

AML comprises multiple entities characterized by specific gene mutations and chromosomal abnormalities that drive leukemogenesis and can be used as prognosticators ${ }^{5,6}$. One of the most frequent chromosomal translocations detected in 5-12\% AML patients is chromosome 16 inversion, $\operatorname{inv}(16)(\mathrm{p} 13 \mathrm{q} 22)$ or $\mathrm{t}(16 ; 16)$ (p13.1;q22) [henceforth inv(16)], which is associated with the FAB M4Eo AML subtype ${ }^{7,8}$. At the molecular level, inv(16) disrupts the $C B F B$ gene encoding the $C B F \beta$ subunit of the corebinding factor $(\mathrm{CBF})$ transcription factor complex that acts as a master regulator of hematopoietic development and lineage specification $^{9,10}$. Specifically, inv(16) breaks and joins $C B F B$ and the smooth-muscle myosin heavy chain (MYH11) gene, creating a fusion gene $C B F B-M Y H 11(\mathrm{CM})$ which encodes a fusion protein $\mathrm{CBF} \beta-\mathrm{SMMHC}^{11}$. Although inv(16) AML patients have a relatively favorable prognosis, only approximately $50-60 \%$ eventually achieve long-term survival with standard chemotherapy ${ }^{12,13}$. Therefore, the development of therapies capable of targeting LSCs is necessary to achieve a cure in the vast majority of the patients.

MicroRNAs (miRNAs) are small non-coding RNA molecules that modulate multiple targets by promoting mRNA degradation or repressing translation ${ }^{14,15}$. MiRNAs are transcribed as primary (pri)-miRNAs, processed into precursor (pre)-miRNAs by DROSHA, exported into the cytoplasm via the RAN (a small GTP-binding RAS-related nuclear protein)-Exportin-5 (XPO5) complex $^{16,17}$, and processed by Dicer into mature miRNAs ${ }^{18}$. In hematopoiesis, miRNAs play critical roles in coordinating context-dependent differentiation programs and cell fate decisions ${ }^{19}$. MiR-126 is highly expressed in endothelial cells and plays a key role in angiogenesis, vascular development and homeostasis ${ }^{20,21}$. Within the hematopoietic compartment, miR126 expression is enriched in long-term hematopoietic stem cells (HSCs) and plays a pivotal role in restraining cell cycle progression and maintaining HSC quiescence ${ }^{22}$. Aberrant miRNA expression profiles have been associated with malignant transformation and prognosis in leukemia and other hematologic malignancies ${ }^{23-27}$. Notably, miR-126 is aberrantly expressed in CBF leukemias including $\mathrm{t}(8 ; 21)$ and $\operatorname{inv}(16)^{25,26}$. We and others have reported that miR-126 contributes to LSC activity, maintenance, and drug resistance in myeloid leukemias ${ }^{28-30}$. Furthermore, we recently reported that activating tyrosine kinase mutations (e.g., BCR-ABL, FLT3-ITD) deregulate intronic miRNA (e.g., miR-126) biogenesis by interfering with RANXPO5 mediated pre-miRNA processing via phosphorylation of SPRED1, a member of the Sprouty family of proteins ${ }^{30,31}$.

Here, we investigated the mechanism and functional impact of miR-126 dysregulation in inv(16) AML. We utilized the conditional $C b f b-M Y H 11$ knock-in mouse model ${ }^{32,33}$ combined with a miR-126 floxed allele ${ }^{34}$ to determine the function and regulatory mechanism of miR-126 during inv(16)-induced AML development and evaluated the efficacy of targeting LSCs using miRisten, an anti-miR-126 oligonucleotide therapeutic.

\section{Results}

Cbfb-MYH11 (CM) upregulates miR-126 expression in hematopoietic stem and progenitor cells (HSPCs). Previous studies have shown that miR-126 is highly expressed and contributes to the relative quiescence of normal CD34 ${ }^{+} \mathrm{HSCs}^{22,28-30}$. Herein we show that $\mathrm{CD}^{+} 4^{+}$cells from inv(16) AML patients have even higher miR-126 levels than $\mathrm{CD}^{+}{ }^{+}$cells from normal healthy donors (Fig. 1a). MiR-126 is located within intron 7 of the EGFL7 gene ${ }^{35}$, which was highly co-expressed with miR-126 (Fig. 1b).

We previously generated a conditional CM knock-in $\left(C b f b^{56 M /+}\right.$ /Mx1-Cre) mouse model ${ }^{32,33}$, which recapitulates human $\operatorname{inv}(16)$ AML development in an average of 3-4 months after induction of $\mathrm{CM}$ expression by poly(I:C) treatment. In a time-sequential analysis of miR-126 levels in mononuclear cells from peripheral blood (PB) of $C M$ knock-in mice, we observed a progressive increase of both miR-126 and Egfl7 levels over time (Fig. 1c, d) and in multiple CM HSPC populations ${ }^{33,36,37}$ (Fig. 1e). Significant upregulation of miR126 was detected as early as 1 month after CM induction (Supplementary Fig. 1a).

CM upregulates Egfl/miR-126 transcription in concert with Gata2. To determine whether CM directly dysregulates miR-126 expression, we forced CM or wild-type CBF $\beta$ expression in $32 \mathrm{D}$ myeloblast cells via MSCV-IRES-GFP (MIG) retroviral transduction. We observed upregulated miR-126, pri-miR-126 and pre-miR-126 as well as Egfl in CM-transduced $\mathrm{GFP}^{+}$cells compared to $C B F B$-transduced $\mathrm{GFP}^{+}$cells (Fig. 1f). Conversely, shRNA-mediated CM knockdown resulted in significant downregulation of miR-126, pri-miR126, pre-miR-126 and Egfl7 in 32D-CM cells (Fig. 1g). We and others have shown that CM induces upregulation of the Gata2 transcription factor ${ }^{33,38}$, which reportedly transactivates the EGFL7/miR-126 promoter ${ }^{39,40}$. Accordingly, we observed increased Gata2 levels upon CM transduction in 32D cells (Supplementary Fig. 1b), as well as in CM knock-in PB (Supplementary Fig. 1c) along with miR-126/ Egfl7 levels (Fig. 1c, d), and AML cells from CM knock-in mice (Fig. 1h). Conversely, Gata2 knockdown in CM knock-in AML cells resulted in downregulation of miR-126 (Fig. 1I, j) and Egfl7 (Supplementary Fig. 1d). Analysis of the promoter regions of both murine and human EGFL7 identified several putative CBF binding sites (TRANSFAC database ${ }^{41}$ ) nearby the GATA-binding sites (Supplementary Fig. 1e). Chromatin immunoprecipitation (ChIP)-qPCR showed that both $\mathrm{CBF} \beta$ and $\mathrm{CM}$ protein indeed could occupy these $\mathrm{CBF}$ sites within the $E g f l 7$ promoter region and that some of the nearby GATA sites were also occupied by GATA2 (Supplementary Fig. 1e). In addition, we confirmed RUNX1, CBF $\beta / C M$ occupancy on the reported RUNX1 site ${ }^{42,43}$ as well as GATA2 binding to the reported GATA2 site ${ }^{42,43}$ in the human EGFL7 promoter by ChIP-qPCR using primary inv(16) AML CD34 ${ }^{+}$cells (Supplementary Fig. 1f). We then performed luciferase reporter assays to evaluate transactivation of the human EGFL7 promoter ${ }^{44}$ by GATA2 and the CBF complex, which is consisted of RUNX1/CBF $\beta$ or RUNX1/CM. A significant increase of EGFL7-luciferase transactivation was observed with forced expression of GATA2, RUNX1/CBF $\beta$ and RUNX1/CM (Fig. 1k). Co-transfection of RUNX1/CBF $\beta$ or RUNX1/CM with GATA2 further increased EGFL7 promoter transactivation, suggesting a co-regulatory activity of GATA2 and RUNX1/CBF on EGFL7/miR-126 transcription.

$\mathrm{CM}$ increases biogenesis of mature miR-126 through the HDAC8/RAN-XPO5-RCC1 axis. Next, we tested whether additional mechanisms other than transcriptional regulation of EGFL7/miR-126 were active in inducing high levels of mature miR-126 in CM cells. To this end, we previously reported and confirmed that CM interacts and enhances the activity of a class I histone deacetylase (HDAC), HDAC8 ${ }^{45}$ (Supplementary Fig. 2a). 
a

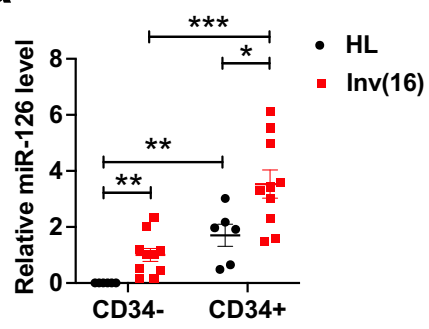

d
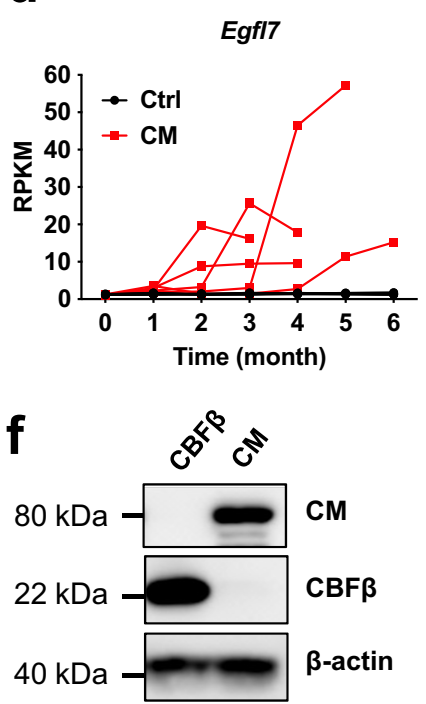

CM

CBF $\beta$

$\beta$-actin

9

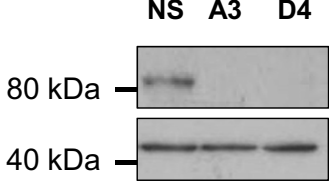

CM

$\beta$-actin
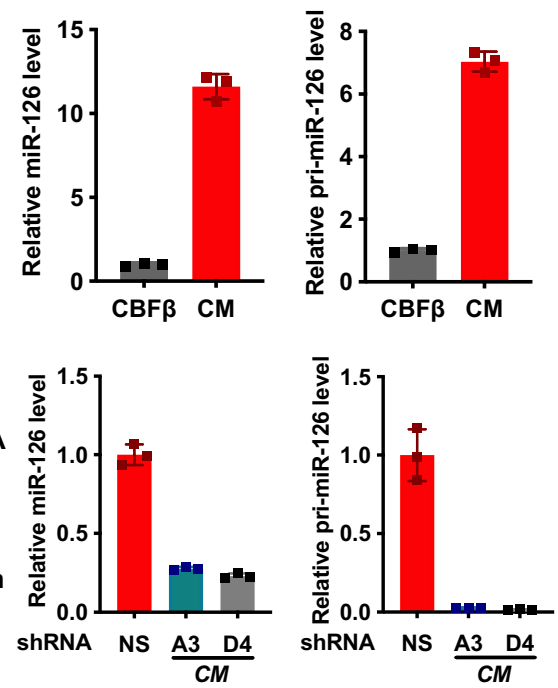

h

i
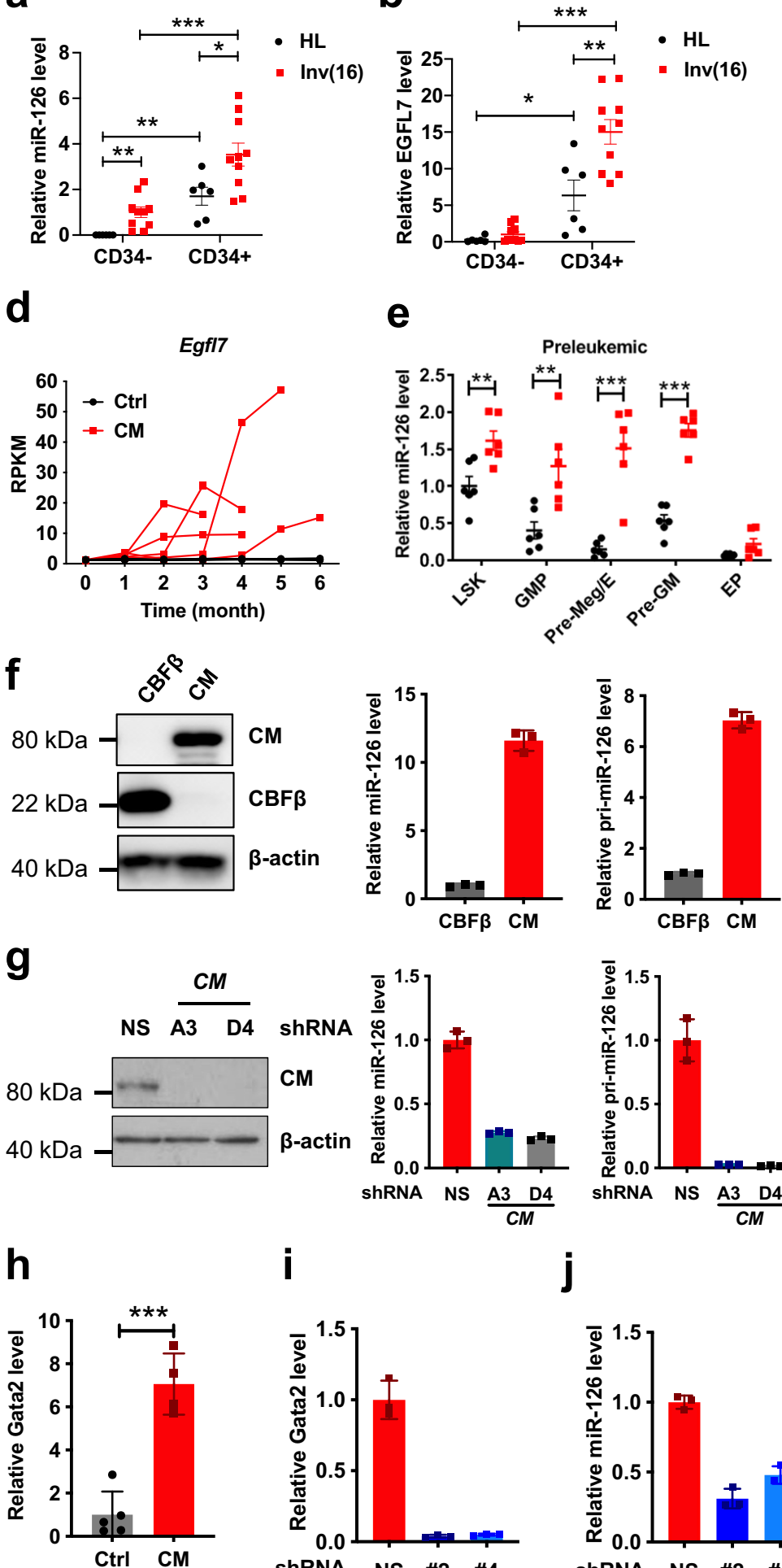

b
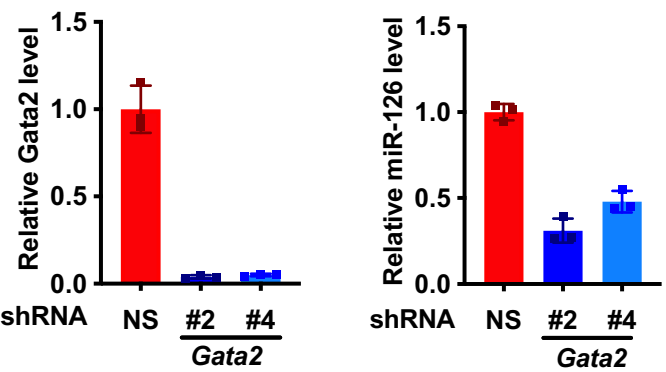
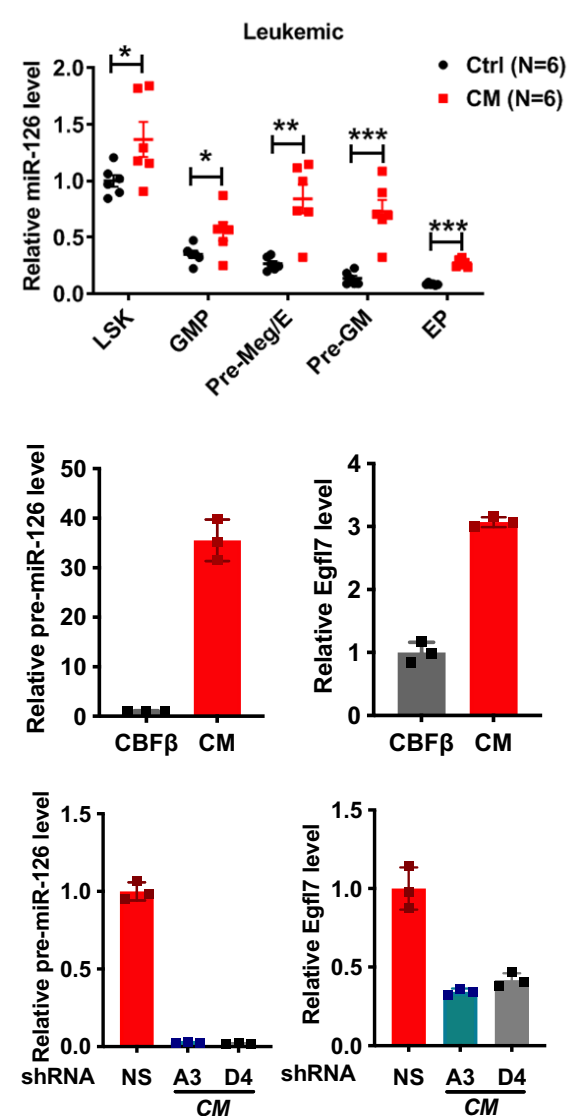

C

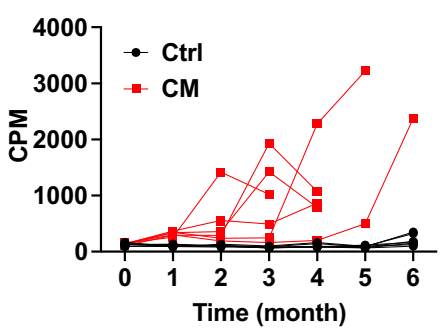

\section{k}

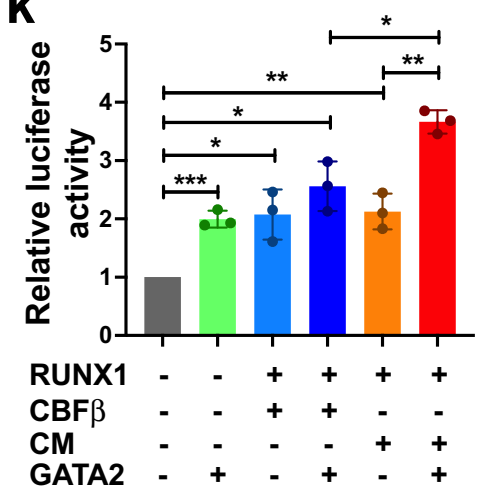

Notably, HDAC8 overexpression (OE) in 32D cells significantly increased mature miR-126 levels while reducing the pre-miR-126 precursor levels compared to vector alone (Supplementary Fig. 2b). Conversely, Hdac8 knockdown or treatment with an HDAC8-selective inhibitor (HDAC8i; 22d) significantly downregulated the levels of mature miR-126 and increased those of pre-miR-126 (Fig. 2a, b). These results led us to postulate a possible regulatory role of $\mathrm{CM}$ in enhancing the miR-126 biogenesis via HDAC8.

First, we tested the impact of CM on HDAC 8 protein stability. We found that CM expression enhanced the half-life of HDAC8 protein following cycloheximide treatment (Supplementary Fig. 2c). HDAC8 protein is subjected to MDM2-mediated ubiquitination (Supplementary Fig. 2d), which was reduced by 
Fig. 1 Cbfb-MYH11 (CM) upregulates miR-126 expression in HSPC. a Relative levels of miR-126 in CD34+ and CD34- cells from healthy (HL; black circle; $n=6 ;{\mathrm{HL} \mathrm{CD} 34^{+}}^{+}$vs. $\left.\mathrm{HLCD} 34^{-} p=0.0036\right)$ donors and inv(16) $\mathrm{AML}$ patients (red square; $n=10$; inv(16) $\mathrm{CD} 34^{+}$vs. $\mathrm{HL} \mathrm{CD34}^{+} p=0.024 ;$ inv(16) CD34- vs. HL CD34- $p=0.0053$; inv(16) CD34+ vs. inv(16) CD34- $p=0.0002$ ). b Relative levels of EGFL7 in CD34+ and CD34- cells from HL donors

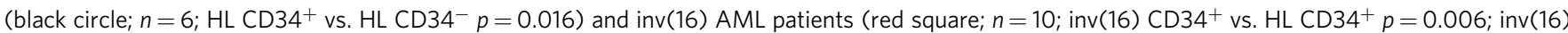
$\mathrm{CD}_{4}{ }^{+}$vs. inv(16) CD34- $\left.p<0.0001\right)$. c Counts per million (CPM) reads for miR-126-3p in PB based on miRNA-seq of control (Ctrl; black line; $\left.n=7\right)$ and CM (red line; $n=6$ ) mice over time until moribund with leukemia (two-way ANOVA analysis showed CM vs. Ctrl $p<0.0001$ ). d Normalized reads per kilobase per million of transcript (RPKM) for Egfl7 in PB based on RNA-seq of Ctrl (black line; $n=7$ ) and CM (red line; $n=6$ ) mice over time (two-way ANOVA analysis showed Ctrl vs. CM $p=0.0003$ ). e Relative levels of miR-126 in CM (red square) preleukemic ( 6 weeks after induction; left; $n=6)$ or leukemic (right; $n=6$ ) vs. Ctrl (black circle; $n=6$ ) HSPC populations, including LSK (preleukemic $p=0.0074$; leukemic $p=0.049$ ), GMP (preleukemic $p=0.0064$; leukemic $p=0.043$ ), Pre-Meg/E (preleukemic $p=0.00015$; leukemic $p=0.0012$ ), Pre-GM (preleukemic $p<0.0001$; leukemic $p=0.0002$ ), and EP (leukemic $p<0.0001$ ). $\mathbf{f}$ Western blot for CBF $\beta$ and CM using anti-CBF $\beta$ in 32D cells transduced with CBFB vs. CM (left). Relative levels of primary pri-miR-126, precursor pre-miR-126, mature miR-126, and Egfl7 in 32D cells expressing CM (red dots/bars) vs. CBF $\beta$ (black dots, gray bars). $\mathbf{g}$ Western blot analysis for CM in 32D-CM non-silencing (NS) control vs. CM shRNA (A3, D4) cells (left). Relative levels of mature miR-126, pri-miR-126, pre-miR-126 and Egfl7 expression in NS (red), CM shRNA-A3 (blue) or CM shRNA-D4 (gray) cells (right). h Relative Gata2 expression in BM samples of control (black/gray; $n=5)$ vs. CM leukemia (red; $n=4 ; p=0.0002$ ) mice. i Relative level of Gata2 in leukemia BM cells transduced with NS control (red), Gata2 shRNA \#2 (dark blue), Gata2 shRNA \#4 (light blue). j Relative expression of miR-126 in leukemia BM cells transduced with NS control (red), Gata2 shRNA \#2 (dark blue), Gata2 shRNA \#4 (light blue). k EGFL7 promoter-firefly luciferase reporter activity normalized with renilla luciferase as internal control in $293 \mathrm{~T}$ cells co-transfected with or without GATA2, RUNX1, CBF $\beta$ or CM expression vectors as indicated (gray: none; green: GATA2; light blue: RUNX1 + CBF $\beta$; dark blue: RUNX1 + CBF $\beta+$ GATA2; orange: RUNX1 + CM; Red: RUNX1 + CM + GATA2). Each dot represents results from an individual sample and data are presented as the mean $\pm S E M$ in $\mathbf{a}, \mathbf{b}, \mathbf{e}, \mathbf{h}$; each line represents the trajectory of an individual mouse in $\mathbf{c}$, $\mathbf{d}$. Representative data of at least three independent experiments are shown in $\mathbf{f}, \mathbf{g}, \mathbf{i}, \mathbf{j}$ and data are presented as the mean \pm SD. Q-PCR data in $\mathbf{a}, \mathbf{b}, \mathbf{e}, \mathbf{f}$ for pri-miR-126, pre-miR-126, Egfl7, and Gata2 were normalized to Beta-2-microglobulin (B2M) and miR-126 data were normalized to RNU44 (human) or snoRNA234 (mouse). The statistical significance for all comparisons shown was determined using two-tailed Student's $T$ tests $\left({ }^{\star} p<0.05 ;{ }^{\star \star} p<0.01 ;{ }^{\star \star \star} p<0.001\right)$.

CM expression (Supplementary Fig. 2e, left) and increased by CM knockdown (Supplementary Fig. 2e, right). ErbB3-binding protein 1 (EBP1) reportedly interacts with another HDAC family member HDAC2 ${ }^{46,47}$. We showed that EBP1 participates in the regulation of HDAC8 ubiquitination as supported by colocalization and physical interaction of HDAC8 and EBP1 in 32D cells (Supplementary Fig. 2f) and reduced MDM2-induced HDAC8 ubiquitination upon EBP1 knockdown (Supplementary Fig. 2g). Forced CM expression significantly decreased the EBP1HDAC8 interaction (Supplementary Fig. 2h, left, top panel), reduced HDAC8 ubiquitination (Supplementary Fig. 2e, left), and enhanced HDAC8 stability (Supplementary Fig. 2h, left, bottom panels). Conversely, CM knockdown rescued these effects (Supplementary Fig. 2e, h, right). Taken altogether, these results support the notion that CM prevents HDAC8-EBP1 binding thereby stabilizing and protecting HDAC8 protein from degradation.

In canonical miRNA biogenesis, nucleus-to-cytoplasm shuttling and maturation of miRNAs are mediated by a protein complex comprising RAN, XPO5, and regulator of chromosome condensation 1 (RCC1) ${ }^{16,48}$. We previously reported that pre-miR-126 transport is mediated by the activity of the RAN-XPO5-RCC1 complex ${ }^{30}$. Utilizing immunofluorescence (IF) and IP assays, we observed colocalization and interaction of HDAC8 with RAN (Fig. 2c). Acetylation of RAN protein reportedly interferes with RAN-RCC1 binding, RAN-GTP/GDP cycles, and RAN function in nucleus-cytoplasm transport ${ }^{49}$. Thus, we hypothesized that the interaction of HDAC8 with RAN leads to deacetylation of RAN and enhanced activation of the nucleus-cytoplasm transportation of pre-miR-126, which results in high levels of mature miR-126. Consistent with our hypothesis, we showed that HDAC8 inhibition with HDAC8i (22d) treatment in 32D-CM cells led to RAN hyperacetylation (Fig. 2d, left, first panel) and decrease of RAN-RCC1 binding (Fig. 2d, left, second panel), RAN-GTP level (Fig. 2d, right), RAN-XPO5 binding (Fig. 2d, left, third panel), and mature miR-126 levels (Fig. 2b). CM knockdown in 32D-CM cells showed similar effects (Fig. 2e). Conversely, CM expression stabilized HDAC8 and in turn increased RAN deacetylation, RAN-GTP level, and RAN-XPO5-RCC1 binding in CM mouse BM cells (Fig. 2f).
Taken altogether, these results indicate that CM upregulates miR-126 expression through multiple regulatory mechanisms of miR-126 transcription and biogenesis (Fig. 2g).

MiR-126 is critical for initiating and maintaining CM-driven AML. To further investigate the contribution of miR-126 to CM-driven leukemogenesis in vivo, we generated mice with conditional $C M$ knock-in and $m i R-126$ floxed alleles $\left(C b f b^{56 M} /\right.$ $\left.+/ m i R-126^{\text {flox/flox} / M x 1-C r e ; ~ C M / m i R-126 ~} 6^{\Delta / \Delta}\right)$. CM expression and miR-126 deletion in CM/miR-126 $6^{\Delta / \Delta}$ mice (Fig. $3 \mathrm{a}, \mathrm{b}$ ) were then induced with poly(I:C) as previously described ${ }^{30,32-34}$. Deletion of miR-126 alone did not alter the immunophenotype or frequencies of HSPC populations (Supplementary Fig. 3a-d). We previously reported that $\mathrm{CM}$ expression leads to expansion of multiple BM HSPC populations preceding the development of leukemia ${ }^{32,33,50}$. Deletion of miR-126 in CM mice reduced the preleukemic expansion of HSPC populations (Fig. 3c, d and Supplementary Fig. 3a) and reversed the CM-induced stressresistance survival of leukemia-initiating progenitor populations previously defined ${ }^{33}$ (Supplementary Fig. 4). CM/miR-126 $6^{\Delta / \Delta}$ mice $(n=26)$ had a significantly reduced rate of overt AML $(50 \%$ vs. $100 \%$ ) and a significantly extended survival (median survival 354 vs. 110 days; $p<0.0001)$ compared to $C M$ mice $(n=26)$ (Fig. 3e). Transplantation of $C M$ preleukemic BM cells into endothelial cell (EC) $-m i R-126^{\Delta / \Delta}$ or wild type (WT) recipients showed a similar rate of AML development and similar survival time (Supplementary Fig. 5), suggesting that miR-126 expression in HSPCs is critical for initiation of CM-driven AML.

To identify molecular pathways regulated by miR-126, we performed RNA-seq using sorted LSK cells isolated from BM of $C M / m i R-126^{\Delta / \Delta}(n=5)$ and $C M$ mice $(n=5)$. We identified a total of 100 genes differentially expressed (68 genes upregulated and 32 genes downregulated) with at least 1.5 -fold change $(p<0.01)$ in expression levels (Fig. 3f and Supplementary Table 1). Gene set enrichment analysis (GSEA) revealed that MYC and E2F targets, mitotic spindle, reactive oxygen species pathway, oxidative phosphorylation, TNFa signaling, and p53 pathways were differentially regulated in $C M / m i R-126^{\Delta / \Delta}$ compared to $C M$ LSK (Fig. 3g, h; Supplementary Fig. 6; Supplementary Table 2). 
a

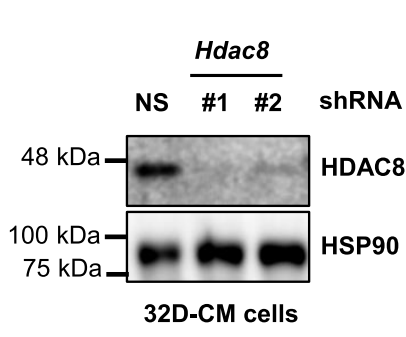

C

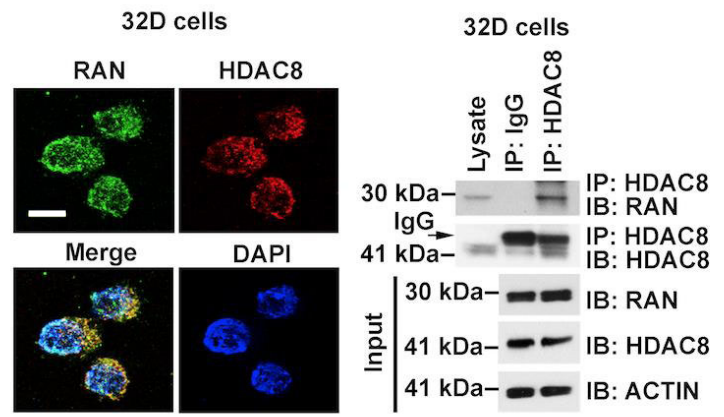

e

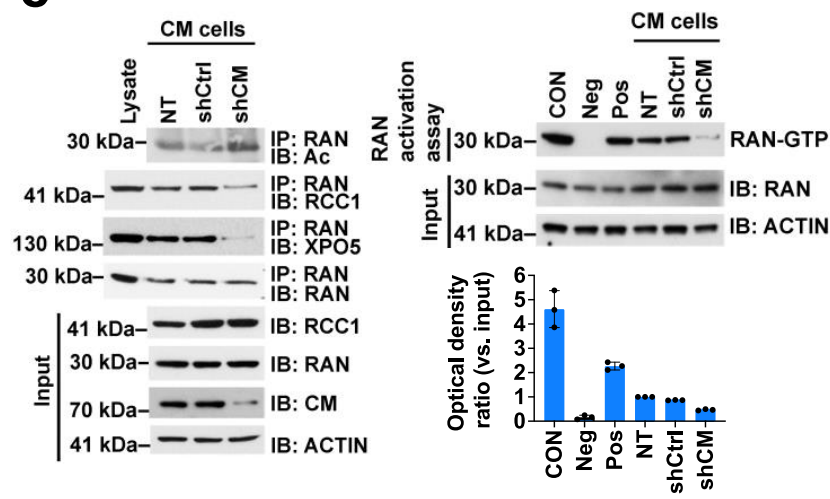

b
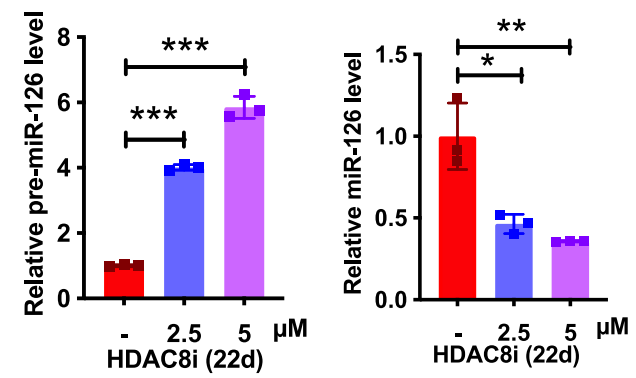

d

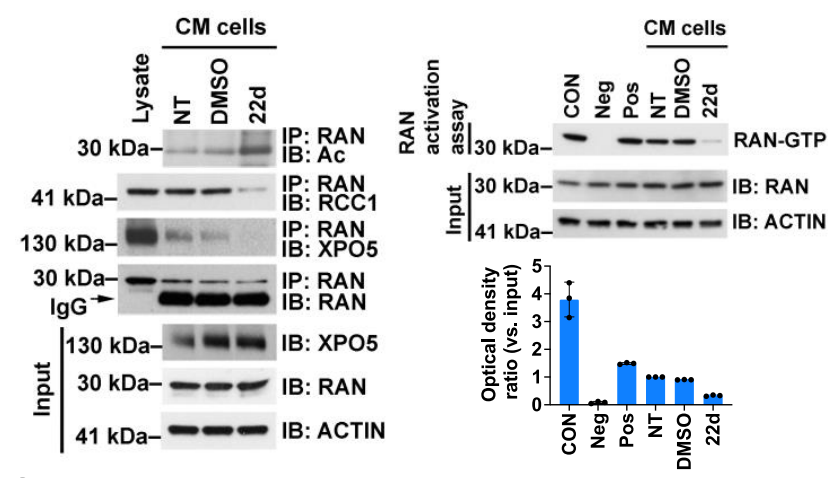

f

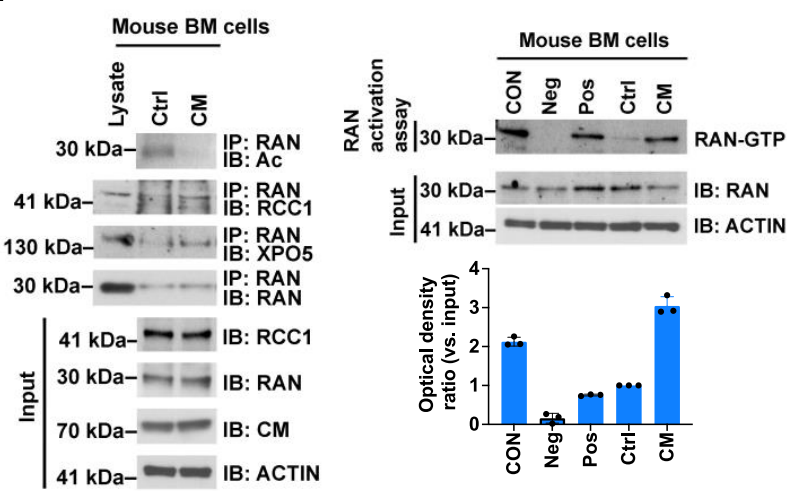

g

Normal

CM
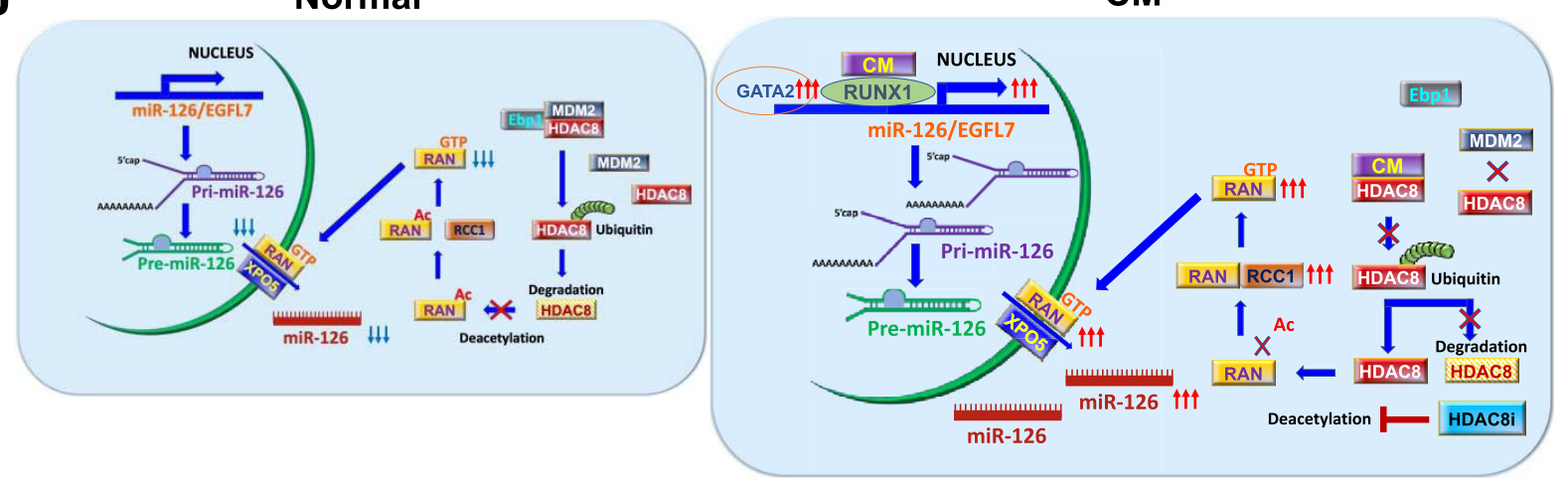

SPRED1 and PLK2 (polo-like kinase 2) are previously reported miR-126 targets $21,23,25,51$ and negative regulators of RAS-MEK-ERK signaling 52,53 , which regulates MYC protein stability by phosphorylation of $\mathrm{S}^{2} 2^{54}$. We verified that both Spred 1 and Plk2 mRNA and protein levels are downregulated in $C M$ LSK and upregulated in CM/miR-126 $6^{\Delta / \Delta}$ LSK cells (Fig. 4 a, b). Consistent with downregulation of Spred1 and Plk2, CM LSK showed increased levels of phosphorylated (p)-ERK, increased p-MYC (S62) and total MYC protein (Fig. 4b). Deletion of miR126 reversed these effects in that increased Spred1, Plk2 in LSK (Fig. 4a), and decreased p-ERK, p-MYC (S62), and MYC protein levels were detected in $C M / m i R-126^{\Delta / \Delta}$ Lin $^{-}$BM cells (Fig. 4c). Similar results were obtained by knocking down miR-126 using a lentiviral vector $\left(\mathrm{GFP}^{+}\right)$expressing miR-126-3p shRNA (shmiR- 
Fig. 2 CM upregulates miR-126 biogenesis through HDAC8/RAN-XPO5 axis. a Western blot analysis of HDAC8 in 32D-CM cells with NS or Hdac8 shRNA (\#1, \#2) (left). Relative expression of pre-miR-126 and mature miR-126 in 32D-CM cells with NS (red) vs. Hdac8 shRNA\#1 (light blue), Hdac8 shRNA\#2 (dark blue) (right). b Relative expression in 32D-CM cells treated with vehicle (red) vs. HDAC8i ( $22 \mathrm{~d} 2.5 \mu \mathrm{M}$ or $5 \mu \mathrm{M}$; purple) for pre-miR-126 (left; $2.5 \mu \mathrm{M} p<0.0001 ; 5 \mu \mathrm{M} p<0.0001$ ) and miR-126 (right; $2.5 \mu \mathrm{M} p=0.012 ; 5 \mu \mathrm{M} p=0.0055$ ). c Representative image of IF co-staining of RAN (green) and HDAC8 (red) in 32D cells (left; scale bar $10 \mu \mathrm{m}$ ). IP with anti-lgG control or anti-HDAC8 followed by immunoblotting (IB) with anti-RAN antibodies (right). Representative results of two independent experiments with similar results are shown. d IP with anti-RAN and IB with antibodies for AcLys, RCC1, XPO5, or RAN in 32D-CM cells not-treated (NT) or treated with DMSO vehicle or 22d ( $2 \mu M$ ) (left). The levels of RAN-GTP determined by RAN activation assay (right). The densitometry of RAN-GTP levels measured from three assays are shown on the bottom. e IP with anti-RAN and IB with antibodies for Ac-Lys, RCC1, XPO5, or RAN (left), and RAN activation assay (right) in 32D-CM cells not-treated (NT) or transduced with shCtrl control or shCM for $24 \mathrm{~h}$. f IP with anti-RAN and IB with antibodies for Ac-Lys, RCC1, XPO5, or RAN (left), and RAN activation assay (right) of BM cells isolated from Ctrl or CM mice. $\mathbf{g}$ Schematic model of the mechanism by which CM regulates miR-126/EGFL7 transcription as well as miR-126 biogenesis through enhancing HDAC8 activity which in turn promotes RAN-XPO5 mediated transportation. Pre-miR-126 was normalized to B2m and mature miR-126 was normalized to snoRNA234. Data are presented as the mean \pm SD and statistical significance shown was determined using two-tailed Student's $T$ tests $\left({ }^{\star} p<0.05 ;{ }^{\star \star} p<0.01 ;{ }^{* \star \star} p<0.001\right)$.

126) (Fig. 4d). In line with downregulation of MYC level, expression of MYC target genes including $E 2 f 1^{55}, C d k 4^{56}$, $N p m 1^{57}$ were significantly reduced (Fig. 4e). These results suggest that miR-126 functions to enhance MYC activity through downregulating SPRED1, PLK2, and activating the ERK-MYC axis.

To validate the leukemogenic role of miR-126 observed in the murine inv(16) AML model, we knocked down miR-126 in human inv(16) AML CD34+ and CD34- cells using shmiR-126 and assessed cell differentiation, proliferation, and apoptosis rates (Supplementary Fig. 7a). With a transduction efficiency of 55-70\% (Supplementary Fig. 7b) and $>70 \%$ miR-126 knockdown in $\mathrm{GFP}^{+}$-transduced cells (Fig. 5a), we detected a significantly increased frequency of more mature $\mathrm{CD} 14^{+}$and $\mathrm{CD} 15^{+}$cell subpopulation compared to control shRNA transduced cells (Supplementary Fig. 7c). In addition, we observed decreased CD34 ${ }^{+}$cell fractions in the $G_{0}$ phase and increased in the $G_{1}$ and $\mathrm{S} / \mathrm{G}_{2} / \mathrm{M}$ phase (Fig. 5b and Supplementary Fig. $7 \mathrm{~d}$ ). Annexin V/ DAPI staining showed increased spontaneous apoptosis rate after miR-126 knockdown (Fig. 5c, d). We verified that miR-126 knockdown resulted in upregulation of SPRED1, PLK2, decreased p-ERK, p-MYC (S62), and MYC levels in primary inv(16) AML CD $34^{+}$cells (Fig. 5e and Supplementary Fig. $7 f-g$ ). MYC plays a crucial role in regulating cell survival ${ }^{58}$ and repression of MYC is known to induce apoptosis in inv(16) AML cells ${ }^{59}$. In line with this, miR-126 knockdown resulted in significant downregulation of anti-apoptotic gene $B C L-2$ and upregulation of pro-apoptotic genes $B A X, B A K$ (Fig. 5e, g). Consequently, we observed increased cleavage of PARP and Caspase 3 (Fig. 5f) and DNA fragmentation (Supplementary Fig. 7h) in miR-126 knockeddown inv(16) AML CD34+ cells. Taken together, these results indicate that miR-126 knockdown resulted in a loss of quiescence, enhanced differentiation, and increased apoptosis of inv(16) AML CD $34^{+}$cells.

Anti-miR-126 therapy inhibits leukemia progression and leukemia-initiating activity of LSCs. Next, to test whether therapeutic targeting of aberrantly expressed miR-126 in CMAML could have an anti-leukemic effect, we used a CpG-antimiR-126 oligonucleotide inhibitor (miRisten) we previously designed $^{30}$. First, we confirmed efficient uptake $(>95 \%)$ in CM AML bulk and LSK cells (Fig. 6a, b) and effective downregulation $(>90 \%)$ of miR-126 after miRisten treatment in vitro (500 $\mathrm{nM}$ and $1 \mu \mathrm{M}$ ) (Fig. 6c). We observed decreased $G_{0}$ and increased $G_{1}$ cell subfractions after miRisten treatment compared to scramble (SCR) control (Fig. 6d) and increased apoptosis rates in miRistentreated compared to SCR-treated cells (Fig. 6e). Next, we confirmed in vivo uptake of Cy3-miRisten in AML blasts in PB (93.2\%) and $\mathrm{BM}(60 \%) 1 \mathrm{~h}$ after intravenous (i.v.) treatment at
$20 \mathrm{mg} / \mathrm{kg}$ (Fig. 6f, g). To evaluate the effects of miRisten on leukemia progression, we then generated a cohort of CM-AML mice $(n=14)$ that developed AML at a similar time by transplanting CM-AML cells into sublethally irradiated (4.5 Gy) WT recipients. When AML burden (as indicated by circulating $\mathrm{cKit}^{+}$blasts) reached approximately $10 \%$ after 6 weeks, mice were randomized and treated either with miRisten or SCR control [20 mg/kg/dose/day, i.v.] for 21 days and monitored for survival (Fig. 6f). MiRisten-treated mice had a significantly longer survival compared with SCR-treated controls (Fig. $6 \mathrm{~h} ; n=7$ each group; median survival 154 vs. 78 days; $p=0.0013)$. Similar treatment with miRisten in normal WT mice did not cause any detectable changes in hematopoietic cell counts or frequency of lineage populations in the $\mathrm{PB}$ monitored over time (Supplementary Fig. 8), suggesting that miRisten have a selective antileukemia activity.

Similar to murine CM-AML cells, human inv(16) AML cells could efficiently uptake miRisten, effectively knocking down miR126 and resulting in increased apoptosis (Supplementary Fig. 9a-e) and reduced quiescence of inv(16) AML CD34 ${ }^{+}$cells (Supplementary Fig. 9f, g). We confirmed that the effects of miRisten was indeed due to reduction of miR-126 since miRisten treatment in inv(16) AML CD34 ${ }^{+}$cells showed no additional effect when miR-126 was knocked down with shmiR-126 (Supplementary Fig. 9h, i). We also confirmed that miR-126 is highly expressed in $\mathrm{t}(8 ; 21)$ AML CD34 ${ }^{+}$cells, and miRisten treatment similarly increased apoptosis and reduced quiescence of $\mathrm{t}(8 ; 21)$ AML CD34 ${ }^{+}$cells (Supplementary Fig. 10).

We further evaluated the in vivo efficacy of miRisten in an inv(16) AML patient-derived xenograft (PDX) model. Primary inv(16) AML cells were expanded in NSGS mice by transplanting T cell-depleted AML blast samples $\left(1 \times 10^{6}\right)$ into irradiated $(2 \mathrm{~Gy})$ mice via intrafemoral (i.f.) injection. A larger cohort of mice bearing human AML $(n=30)$ was then generated by transplanting human (h) $\mathrm{CD}_{4} 5^{+} \mathrm{BM}$ cells $\left(1 \times 10^{6}\right)$ recovered from the above described PDXs into irradiated (2 Gy) NSGS recipients via intravenous injection (Fig. 7a). These secondary (s) PDXs were then treated with miRisten or SCR control $(20 \mathrm{mg} / \mathrm{kg} /$ dose, i.v., daily) for 3 weeks. At the end of treatment, we observed significantly reduced hCD $45^{+}$ burden in miRisten-treated BM compared to SCR control (Fig. 7a, b, d). Moreover, the $\mathrm{CD} 34^{+} / \mathrm{hCD} 45^{+}$frequency was reduced (Fig. $7 \mathrm{c}, \mathrm{e}$ ) and the $\mathrm{CD} 15^{+} / \mathrm{hCD} 45^{+}$cell frequency was increased in miRisten-treated sPDXs (Supplementary Fig. 11a-b), suggesting that miRisten treatment promoted partial differentiation of primitive inv(16) AML cells. MiRisten-treated sPDX mice $(n=6)$ also lived significantly longer than SCR-treated controls (Fig. 7f; $n=6$; median survival 136 days vs. 117 days; $p=0.016)$. In secondary transplant experiments, the recipients $(n=5)$ of BM cells from miRsten-treated mice had a significantly longer survival than 
a
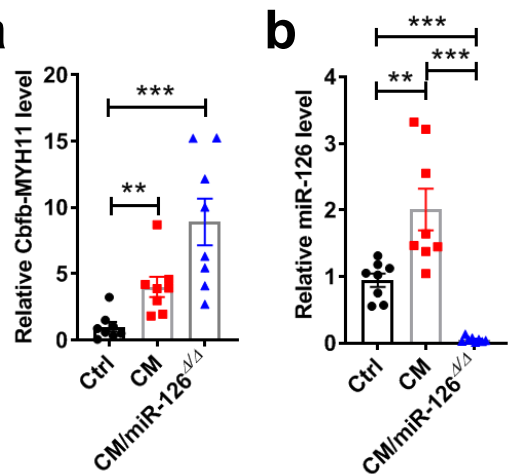

d

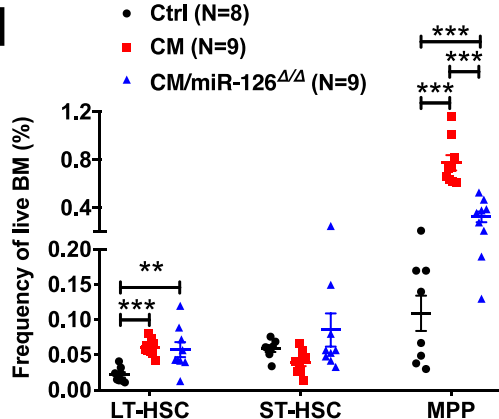

f

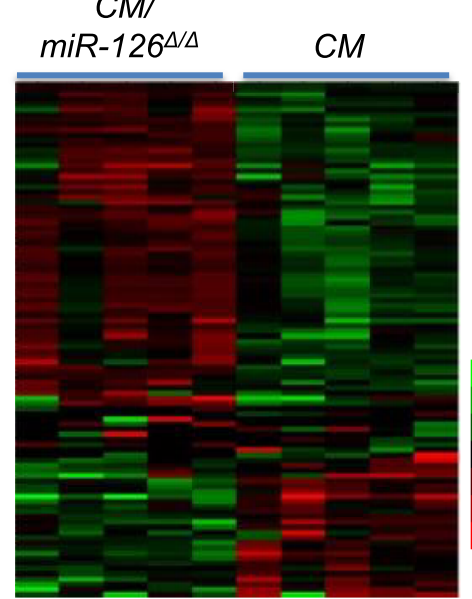

$\mathbf{h}$

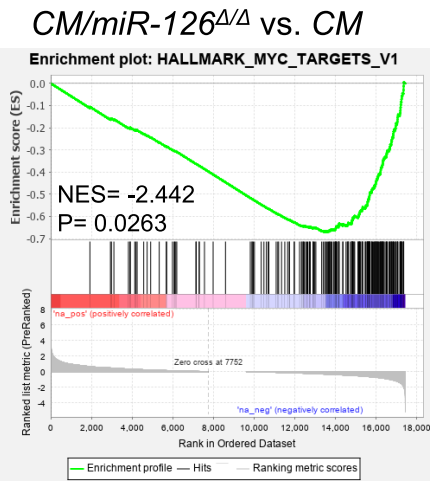

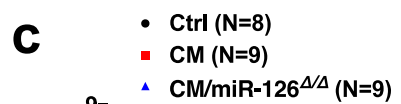
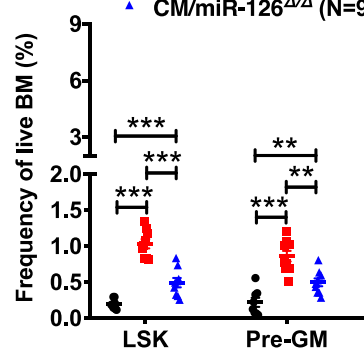

(1)

e

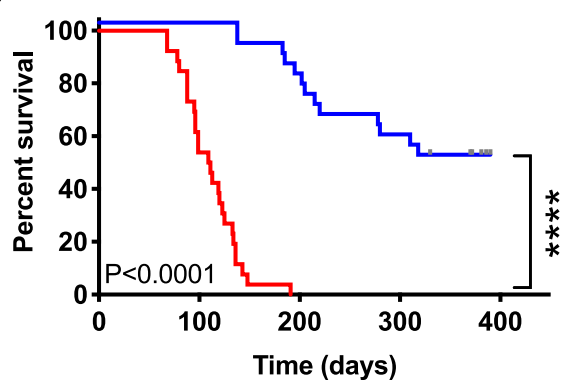

- $\mathrm{CM}(\mathrm{N}=26)$

$-\mathrm{CM} / \mathrm{miR}-126^{\Delta / \Delta}(\mathrm{N}=26)$

g

GSEA: CM/miR-126 $1 / \Delta$ vs. CM LSK

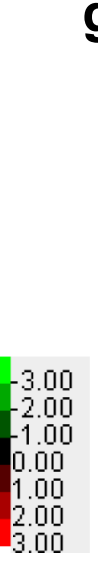

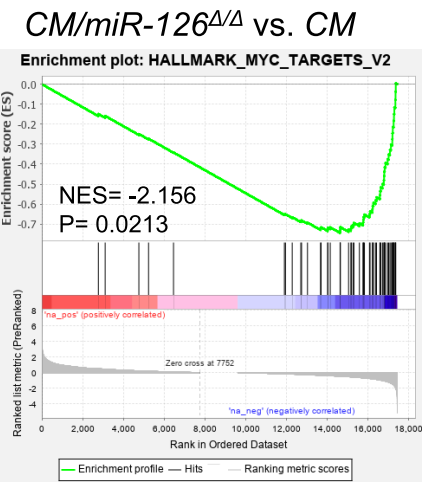

the recipients $(n=5)$ of BM cells from SCR-treated group (Fig. 7g; median survival 199 days vs. 137 days; $p=0.013$ ). Altogether, these results indicate that in vivo administration of miRisten effectively inhibits AML propagation and impedes leukemia-initiating activity of inv(16) AML LSCs.

Given that chemotherapy remains the standard therapy for AML, we then tested the activity of miRisten combined with a chemotherapy “ $5+3$ ” regimen using Ara-C $(50 \mathrm{mg} / \mathrm{kg} / \mathrm{day}$, 5 days) and DNR $(1.5 \mathrm{mg} / \mathrm{kg} /$ day, 3 days $)$ in a CM AML mouse model with a tdTomato ${ }^{+}$Cre-reporter $\left(\mathrm{Cbfb} b^{56 \mathrm{M} /+} / \mathrm{M} \times 1-\mathrm{Cre} /\right.$ td Tomato $^{+}$), which developed transplantable AML 3-6 months after poly(I:C) induction. AML cells from $C b f b^{56 M /+} / M \times 1-C r e /$ tdTomato $^{+}$mice were transplanted into sublethally irradiated syngenic C57/B6 recipients $\left(1 \times 10^{6}\right.$ cells), which were then 
Fig. 3 High miR-126 promotes survival of HSPCs and leukemia-initiation induced by CM. a Relative Cbfb-MYH11 expression in control (Ctrl; black), CM (red; $C M$ vs. Ctrl $p=0.0031$ ), and $C M / m i R-126^{\Delta / \Delta}$ (blue; $C M / m i R-126^{\Delta / \Delta}$ vs. Ctrl $p=0.0006$ ) preleukemic BM cells. b Relative expression levels of miR126 in control (black), CM (red; CM vs. Ctrl $p=0.0062$ ), and CM/miR-126 $4 / \Delta$ (blue; CM vs. CM/miR-126 $\Delta / \Delta p<0.0001, C M / m i R-126^{\Delta / \Delta}$ vs. Ctrl $p<0.0001)$ preleukemic BM cells. c Frequency of LSK (CM vs. Ctrl $p<0.0001 ; C M / m i R-126^{\Delta / \Delta}$ vs. Ctrl $p=0.0009 ; C M$ vs. CM/miR-126 $\left./ \Delta p<0.0001\right)$, myeloid/erythroid progenitor populations including Pre-GM (CM vs. Ctrl $p<0.0001 ; C M / m i R-126^{\Delta / \Delta}$ vs. Ctrl $p=0.0046 ; C M$ vs. $C M / m i R-126^{\Delta / \Delta}$ $p=0.0012)$, GMP (CM vs. Ctrl $p=0.0002 ; C M / m i R-126^{\Delta / \Delta}$ vs. Ctrl $\left.p=0.0043\right)$, Pre-Meg/E (CM vs. Ctrl $p=0.0001 ; C M / m i R-126^{\Delta / \Delta}$ vs. Ctrl $p=0.0028 ; C M$ vs. $\left.C M / m i R-126^{\Delta / \Delta} p=0.012\right), M P\left(C M\right.$ vs. $C \operatorname{trl} p=0.0001 ; C M / m i R-126^{\Delta / \Delta}$ vs. Ctrl $p=0.017 ; C M$ vs. $\left.C M / m i R-126^{\Delta / \Delta} p=0.0079\right)$ in $C \operatorname{trl}$ (black), $C M$ (red) and $C M / m i R-126^{\Delta / \Delta}$ (blue) preleukemic BM. Flow cytometry gating strategies for HSPC populations are shown in Supplementary Fig. $3 \mathrm{~A}$. d Frequency of LT-HSC (CM vs. Ctrl $p<0.0001 ; C M / m i R-126^{\Delta / \Delta}$ vs. Ctrl $\left.p=0.008\right)$, ST-HSC, and MPP (CM vs. Ctrl $p<0.0001 ; C M / m i R-126^{\Delta / \Delta}$ vs. Ctrl $p=0.0009 ; C M$ vs. $C M / m i R-126^{\Delta / \Delta} p<0.0001$ ) subsets in control (black), $C M$ (red), and $C M / m i R-126^{\Delta / \Delta}$ (blue) preleukemic BM. e Kaplan-Meier survival curve of induced $C M$ (red line; $n=26$; median survival 110 days) or $C M /$ miR-126 ${ }^{\Delta / \Delta}$ mice (blue line; $n=26$; median survival 354 days) monitored up to 1 year. The statistical significance was determined using Log-rank (Mantel-Cox) test $(p<0.0001)$. $\mathbf{f}$ The hierarchical clustering heatmap is composed of log2-based RPKM values of the top 100 differential expression genes $(\geq 1.5$-fold change; $p<0.01)$ identified by RNA-seq analysis of $C M / m i R-126^{\Delta / \Delta}$ LSK $(n=5)$ vs. CM LSK $(n=5)$. The color scale is originated from the "redgreen" palette in Cluster 3.0 software, while the color intensity is related to the range of log2-based RPKM values ( -3 to 3). The quasi-likelihood (QL) F-test (one-sided) with likelihood ratio test is carried out to determine the differentially expressed genes (DEG). The "Benjamini-Hochberg" approach is implemented for calculating adjusted $p$ values in multiple comparisons. $\mathbf{g}$ Normalized enrichment score (NES) plot for top 10 most positively and negatively enriched Hallmark Signature pathways identified by GSEA. $\mathbf{h}$ Enrichment plots for most significantly enriched pathways (ordered by $p$ value). The enrichment score (ES) is calculated based on the pre-ranked gene list obtained by

" $-\log 10(p$ value for DEG) $\times \operatorname{sign}(\log 2$ (fold change))". Nominal $p$ value (one-sided) is estimated from background ES (derived from the random permutation of genes) by using the positive or negative portion of the distribution for the sign of ES. The adjusted significance of ES is calculated by false discovery rate (FDR) for multiple hypothesis testing. The detailed results for top 13 most enriched pathways are shown in Supplementary Table 2. Q-PCR data for CbfbMYH11 were normalized with Hprt and miR-126 was normalized to SnoRNA234 in a, b. Each dot in a-c represents result from an individual mouse and data are presented as the mean \pm SEM; statistical significance was determined using two-tailed Student's $T$-tests $\left({ }^{\star} p<0.05 ;{ }^{\star \star} p<0.01\right.$; $\left.{ }^{\star \star \star} p<0.001\right)$.

treated with SCR control $(20 \mathrm{mg} / \mathrm{kg} / \mathrm{dose}$, i.v., daily for 3 weeks; $n=10)$, miRisten $(20 \mathrm{mg} / \mathrm{kg} /$ dose, i.v., daily for 3 weeks; $n=8)$, Ara-C + DNR (A + D; “ $5+3$ ”; $n=8)$, or miRisten and A + D (during week $3 ; n=8$ ) combined (Fig. 8a). All treated mice were sacrificed 7 days after the final treatment administration and evaluated for AML burden in $\mathrm{PB}, \mathrm{BM}$, and spleen. MiRisten treatment significantly reduced splenic disease burden (Fig. 8b, c) as well as the frequency of dTomato ${ }^{+}$AML cells in $\mathrm{PB}$, spleen, and BM compared with SCR-treated controls (Fig. 8d-g). Combination of miRisten with $\mathrm{A}+\mathrm{D}$ further decreased disease burden compared to miRisten alone (Fig. 8b-g). The frequency and cell number of BM myeloid progenitor (MP) subpopulations were significantly reduced by miRisten/A $+\mathrm{D}$ combined treatment compared to control or single (miRisten or $\mathrm{A}+\mathrm{D}$ ) treatment (Supplementary Fig. 12). To evaluate LSC activity, we transplanted BM cells from each treated group into secondary recipients $\left(1 \times 10^{6}\right.$ cells; $\left.n=10-11\right)$ and monitored for leukemia onset and survival. White blood cell count (WBC) and flow cytometry performed 7 weeks later showed significantly lower PB AML cell expansion in recipients of miRisten-treated cells compared to the SCR group (Fig. 8h, i). Further reduction was seen in the miRisten/A $+\mathrm{D}$ combined group compared to miRisten or A $+\mathrm{D}$ groups. Notably, transplants of miRistentreated group showed significantly prolonged survival compared to the SCR-treated group (median survival 95.5 vs. 84.5 days; $p=0.0004$ ) while the A + D-treated group showed no improvement of survival (Fig. 8j). Further prolonged survival was seen in transplants of combined miRisten/A $+\mathrm{D}$ treatment compared to the miRisten group (median survival 102 vs. 95.5 days; $p=0.0386$ ). Collectively, these results indicate that systemic miRisten treatment decreased LSC activity in vivo and combination of miRisten with chemotherapy significantly inhibited AML progression and enhanced survival.

\section{Discussion}

We demonstrate here a critical function of miR-126 in initiating and maintaining inv(16) AML by showing that genetic deletion of miR-126 prevented leukemia development in 50\% of CM knockin mice and significantly prolonged their survival. To develop an effective anti-miR-126 therapy, we employed the CpG-anti-
miR126 inhibitor, miRisten ${ }^{30}$, which efficiently depleted miR-126 and significantly reduced AML blasts and leukemia-initiating LSCs both in inv(16) AML murine and PDX models.

High miR-126 was among the most significant miRNA expression signatures of CBF AML, which includes AML with $t(8 ; 21)$ and $\operatorname{inv}(16)^{25,26}$. A previous report by Li et al. ${ }^{60}$ showed that higher miR-126 is associated with poor prognosis in AML patients with $t(8 ; 21)$. However, the functional impact and mechanism of miR-126 dysregulation in inv(16) AML has been unclear. It has been suggested that hypomethylation of $\mathrm{CpG}$ islands in genomic regions hosting miR-126 is associated with increased expression of miR-126 in CBF leukemias ${ }^{60}$. Our studies reveal that miR-126, located within intron 7 of the Egfl7 gene, is upregulated by the CM fusion protein via at least two distinct mechanisms. First, CM upregulates transcription of Egfl7 and miR-126 through increased expression of GATA2, which functions to transactivate EGFL7/miR-126 $6^{39,40}$ ). CBF transcription factors are known to cooperate with a variety of hematopoietic regulators, including GATA2, in transcriptional regulation ${ }^{61}$. In addition, Zhen et al. ${ }^{62}$ recently demonstrated that CM mainly act as a coactivator of RUNX1 target genes during leukemia development. Consistent with this, our results indicate that CM works together with RUNX1 and GATA2 to activate Egfl7/miR-126 transcription. Consistent with a cooperative upregulation of Egfl7/miR-126, knockdown of CM or Gata2 resulted in significant decrease of Egfl7/miR-126 levels. Furthermore, consistent with reduced leukemogenesis upon miR-126 deletion, Gata2 deficiency also significantly delayed CM leukemogenesis ${ }^{38}$. Of note, transcriptional dysregulation of the $E g f l 7 / \mathrm{miR}-126$ promoter is not the only mechanism through which CM increases the level of miR126. In fact, we demonstrated that $\mathrm{CM}$ enhances the biogenesis (i.e., maturation) of miR-126 through a previously unrecognized mechanism involving the HDAC8/RAN-XPO5 axis. We previously reported that $H D A C 8$ is upregulated in inv(16) AML and that CM interacts with HDAC8 and enhances HDAC8-mediated deacetylation of $\mathrm{p} 53^{45}$. We show here that CM-HDAC8 interaction also enhances the stability of HDAC8 by preventing the binding of HDAC8 to EBP1, which otherwise mediates MDM2depedent protein ubiquitination and degradation. We previously reported that pre-miR-126 is exported from the nucleus to the 
a

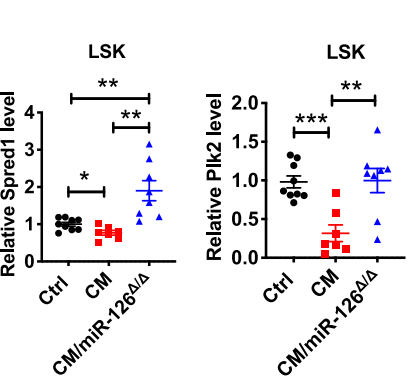

c

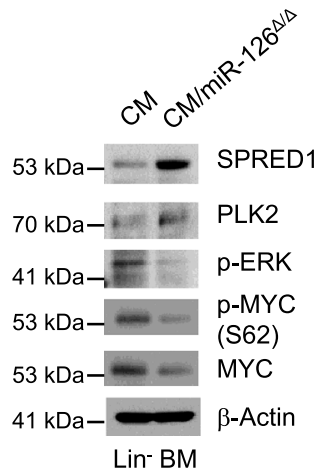

b

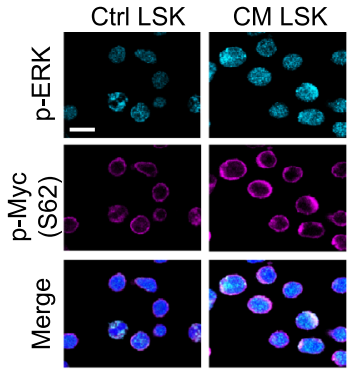

d
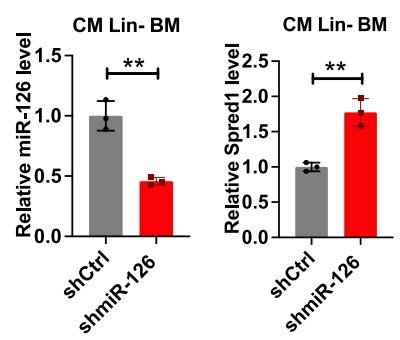

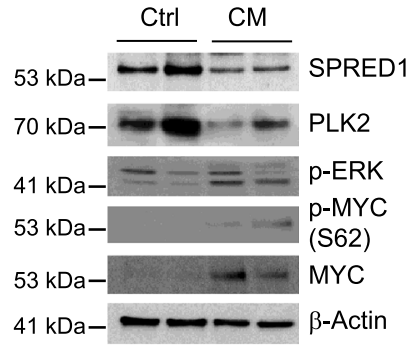

Lin- BM

e
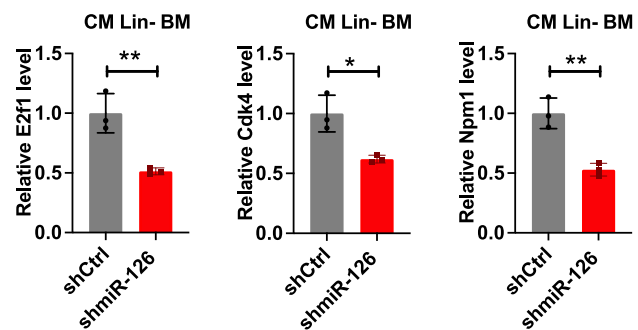

Fig. 4 Expression of miR-126 enhances MYC activity through downregulating SPRED1, PLK2, and activating the ERK-MYC axis in CM-AML. a Relative expression levels Spred1 (CM vs. Ctrl $p=0.015 ; C M / m i R-126^{\Delta / \Delta}$ vs. Ctrl $p=0.0022 ; C M$ vs. $\left.C M / m i R-126^{\Delta / \Delta} p=0.0035\right)$ and $P / k 2(C M$ vs. Ctrl $p=0.0002$; $C M$ vs. $C M / m i R-126^{\Delta / \Delta} p=0.0042$ ) normalized to level of $B 2 m$ in Ctrl (black), $C M$ (red) and $C M / m i R-126^{\Delta / \Delta}$ (blue) preleukemic LSK. b Representative immunostaining of p-MYC (S62; magenta) and p-ERK (cyan) in control and CM AML LSK (left; scale bar $10 \mu$ m); western blot of SPRED1, PLK2, p-ERK, p-MYC (S62), and MYC in control and CM AML lineage-negative ( Lin $^{-}$) BM cells (right). c Western blot of SPRED1, PLK2, p-ERK, p-MYC (S62) and MYC in CM vs. CM/miR-126//A Lin- BM cells. d Relative expression in CM-AML cells expressing shCtrl (black) vs. shmiR-126 (red) for miR-126 ( $p=0.0018)$ and Spred1 ( $p=0.0028$ ) normalized to snoRNA234 and B2m, respectively (left); western blot of SPRED1, p-ERK, p-MYC (S62), and MYC (right) 2 days after transduction with shCtrl or shmiR-126 (right). e Relative expression levels of E2f1 ( $p=0.0072), C d k 4(p=0.0136), N p m 1$ ( $p=0.0041)$ normalized to level of B2m in shCtrl (black) or shmiR-126 (red) expressing CM-AML cells. Each dot represents data from an individual mouse and presented as the mean \pm SEM in $\mathbf{a}$; data in $\mathbf{d}$, e are presented as the mean \pm SD and statistical significance for all comparisons shown was determined using two-tailed $T$ tests $\left({ }^{\star} p<0.05 ;{ }^{\star \star} p<0.01 ;{ }^{\star \star \star} p<0.001\right)$; representative results of two independent experiments with similar results are shown in $\mathbf{b}, \mathbf{c}$.

cytoplasm through a "gatekeeper" RAN-XPO5 complex during miR-126 biogenesis ${ }^{30}$. Here, we discovered that HDAC8 deacetylates and enhances the activity of RAN protein, as supported by the increased binding of RAN to RCC1 and XPO5. This results in increased activity of the "gatekeeper" RAN-XPO5 complex with higher levels of pre-miR-126 transported to the cytoplasm for completion of the last step of miR-126 biogenesis. Although $\mathrm{t}(8 ; 21)$ AML also expresses high levels of miR-126 (Supplementary Fig. 10a), EGFL7, GATA2, and HDAC8 levels were not significantly increased (Supplementary Fig. 10f), suggesting that miR-126 upregulation likely occurs via alternative mechanism(s). This and our recent reports highlight how distinct mechanisms of miR-126 dysregulation driven by specific leukemogenic mutations (i.e., CBFB-MYH11, BCR-ABL, FLT3-ITD) play a relevant role in leukemia pathobiology $y^{30,31}$. Furthermore, the HDAC8/ RAN-XPO5 axis we discovered here can potentially contribute to
miR-126 dysregulation in other subsets of leukemia or other cancer types with aberrant HDAC8 activity.

We and others have reported that high expression of miR-126 is an LSC-associated signature and a determinant of LSC stemness in acute and chronic myeloid leukemias ${ }^{29,30}$. By promoting cell cycle quiescence, miR-126 supports the maintenance and drug resistance of LSCs. On the other hand, forced expression of miR-126 in HSPC led to oncogenic transformation with features of $\mathrm{B}$ cell acute lymphoblastic leukemia ${ }^{63}$. In addition, Li et al. reported that overexpression and knockout of miR-126 both promote leukemogenesis induced by $t(8 ; 21)^{60}$. Our genetic studies revealed that aberrantly expressed miR-126 plays a critical role in initiating leukemogenesis and maintaining AML induced by inv(16) generated CM fusion protein. We and others have shown that CM expression impairs multi-lineage differentiation and induces expansion of preleukemic stem cells and progenitor 
a

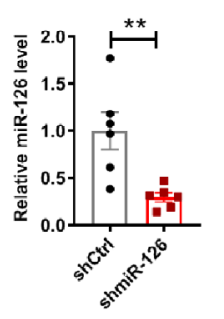

C

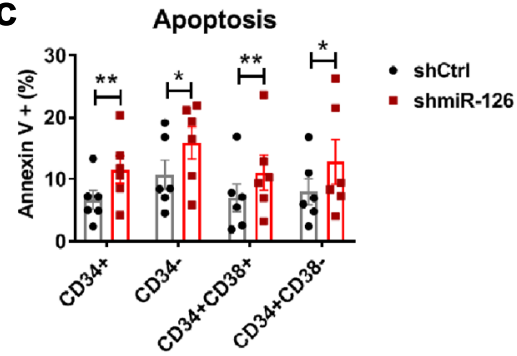

e

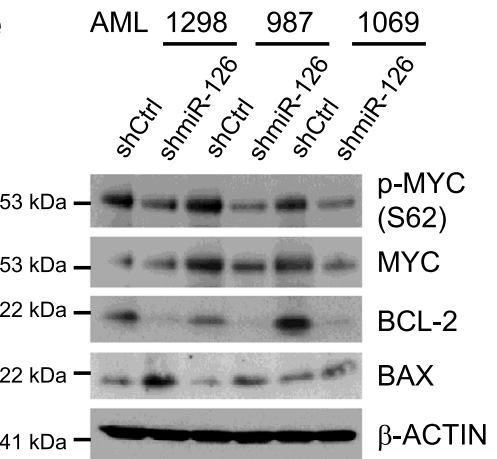

b

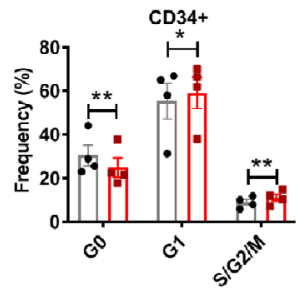

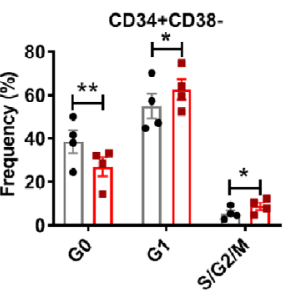

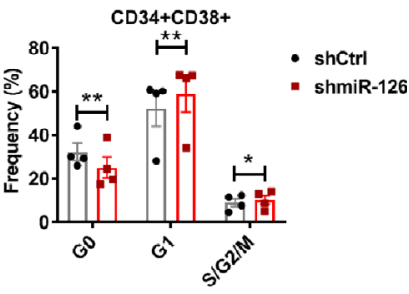

d

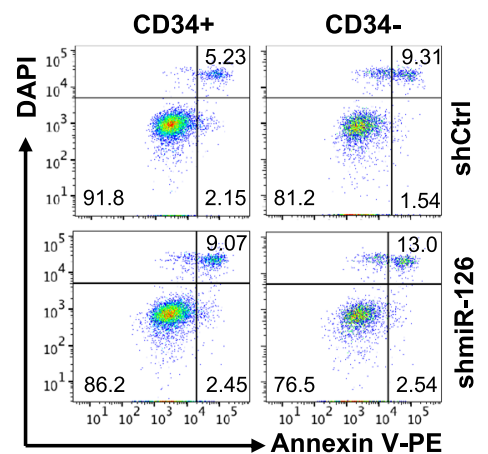

f

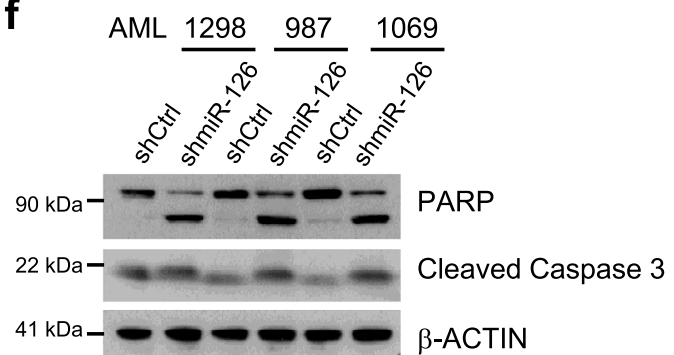

g
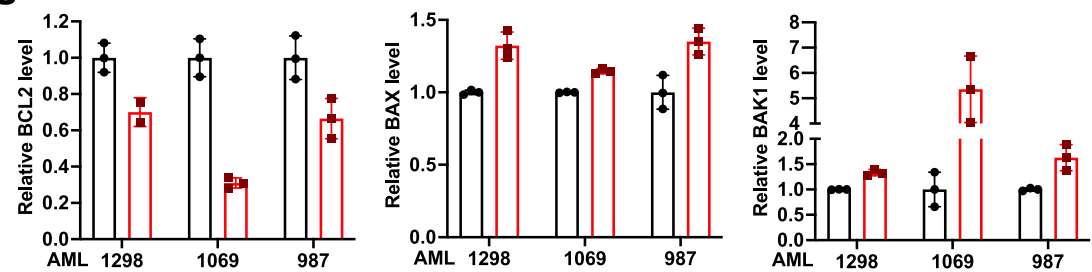

- shCtrl

- shmiR-126

Fig. 5 Knockdown of miR-126 promotes apoptosis and reduces quiescence of primitive inv(16) AML cells. a Relative levels of miR-126 in inv(16) AML $\mathrm{CD} 34^{+}$cells transduced with shCtrl (black) or shmiR-126 (red; $p=0.006$ ), as assessed by qPCR and normalized with internal levels of RNU44. $\mathbf{b}$ Frequency (\%) of $\mathrm{G}_{0}, \mathrm{G}_{1}$ or $\mathrm{S} / \mathrm{G}_{2} / \mathrm{M}$ phases of cell cycle in shCtrl (black) or shmiR-126 (red) transduced inv $(16) \mathrm{AML}_{\mathrm{CD}} \mathrm{CD}^{+}\left(\mathrm{G}_{0} p=0.0073 ; \mathrm{G}_{1}\right.$ $\left.p=0.046 ; \mathrm{S} / \mathrm{G}_{2} / \mathrm{M} p=0.0094\right), \mathrm{CD}_{4}{ }^{+} \mathrm{CD}_{3} 8^{-}\left(\mathrm{G}_{0} p=0.0069 ; \mathrm{G}_{1} p=0.0184 ; \mathrm{S} / \mathrm{G}_{2} / \mathrm{M} p=0.0138\right)$, and $\mathrm{CD}_{34}{ }^{+} \mathrm{CD}_{3} 8^{+}\left(\mathrm{G}_{0} p=0.0072 ; \mathrm{G}_{1}\right.$ $\left.p=0.0015 ; \mathrm{S} / \mathrm{G}_{2} / \mathrm{M} p=0.0259\right)$ populations. c Frequency (\%) of apoptotic cells defined by Annexin $\mathrm{V}+$ in shCtrl (black) vs. shmiR-126 (red) transduced

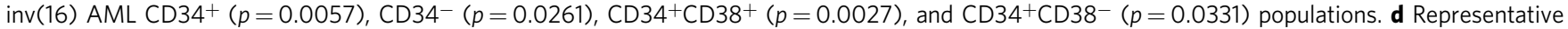
FACS plots showing gating strategy and frequency of Annexin V/DAPI staining in CD34 ${ }^{+}$and CD34- fractions of inv(16) AML samples transduced with shCtrl or shmiR-126. e Western blot of p-MYC (S62), MYC, BCL2, BAX in CD34+ cells from inv(16) AML patients (AML1298, 987, 1069) 2 days after transduction with shCtrl or shmiR-126. f Western blot of PARP and Cleaved Caspase 3 in CD34+ cells from inv(16) AML patients (AML1298, 987, 1069) 2 days after transduction of shCtrl or shmiR-126. $\mathbf{g}$ Relative levels of $B C L 2, B A X, B A K 1$ in CD34+ cells from inv(16) AML patients (AML\#1298, 1069, 987) transduced with shCtrl (black) or shmiR-126 (red), as assessed by qPCR and normalized using internal levels of $B 2 M$. Data are presented as the mean \pm SD. Two-way ANOVA showed $p<0.0001$ for $B C L 2 ; p<0.0001$ for $B A X$, and $p=0.006$ for BAK1. In a-c, each dot represents result from an individual patient and data are presented as the mean \pm SEM; statistical significance shown was determined using two-tailed Student's $T$ tests $\left({ }^{\star} p<0.05\right.$; ${ }^{\star \star} p<0.01$; ${ }^{\star \star \star} p<0.001$ ). Representative of two independent experiments with similar results are shown in $\mathbf{e}, \mathbf{f}$.

cells; however, additional mutations are required for malignant transformation $32,50,64-66$. These aberrantly expanded CM preleukemic HSPCs display enhanced survival and serve as cellular reservoirs susceptible to acquisition of additional alternations needed for further clonal evolution ${ }^{33}$. Deletion of miR-126 significantly increases apoptosis especially in the LSK and
Pre-Meg/E immunophenotypic subsets (Supplementary Fig. 3), which we previously reported are the leukemia-initiating populations prone to malignant transformation in $\mathrm{CM}$ knock-in mice $^{33}$. MiR-126 deletion in CM LSK alters expression of multiple pathways important for malignant transformation or AML maintenance, highlighting the multifaceted impact of miR-126. 
a

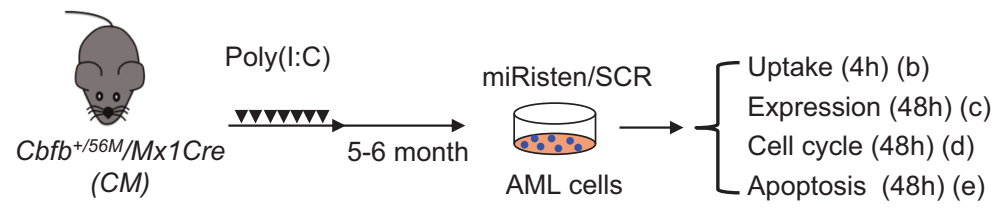

b

miRisten/vehicle uptake in vitro

C
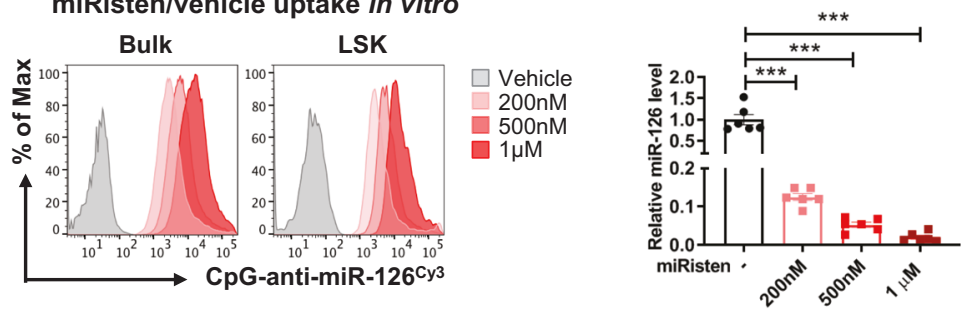

d

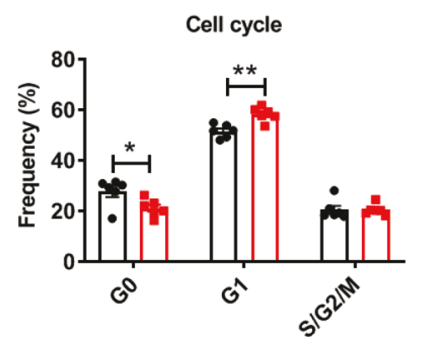

e

SCR

- miRisten

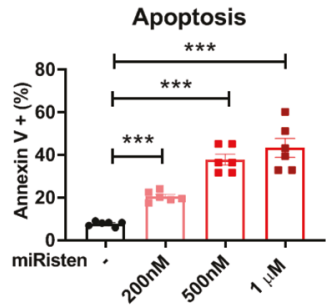

f

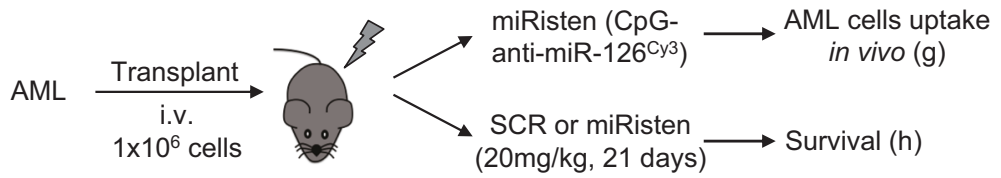

g

miRisten/vehicle uptake in vivo
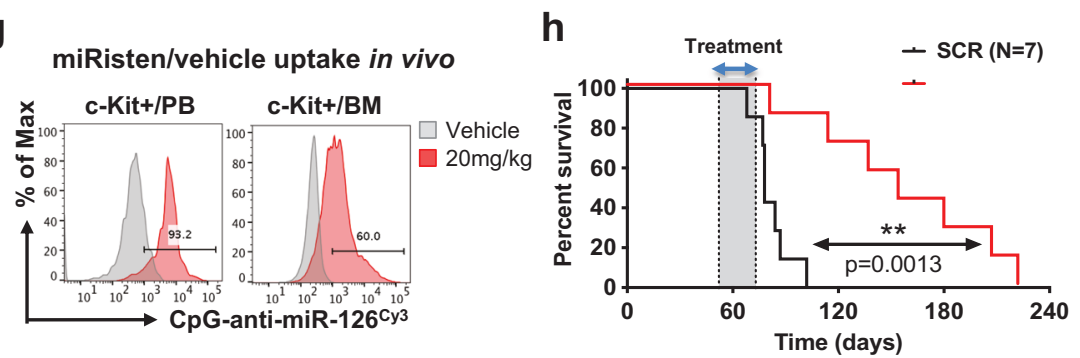

Fig. 6 Targeting miR-126 by miRisten increases AML cell apoptosis and delays CM-AML progression. a Schematic of experimental design. AML cells isolated from CM induced leukemia mice were treated with miRisten or SCR control ex vivo and assessed for uptake, miR-126 expression, cell cycle, and apoptosis at various time points. b The frequency of uptake for miRisten (CpG-anti-miR-126cy3; red) or vehicle (gray) in bulk or LSK cells from CM leukemia BM. c Relative levels of miR-126 in CM AML cells after treatment with SCR control (black) or miRisten $(200 \mathrm{nM}, 500 \mathrm{nM}, 1 \mu \mathrm{M}$; pink/red; $p<0.0001$ for all doses) as determined by qPCR and normalized to SnoRNA234 expression. $\mathbf{d}$ Frequency (\%) of $\mathrm{G}_{0}, \mathrm{G}_{1}$, or $\mathrm{S} / \mathrm{G}_{2} / \mathrm{M}_{\mathrm{m}}$ phases of cell cycle in CM AML cells after treatment with SCR control (black) or miRisten (red; $\mathrm{G}_{0} p=0.031 ; \mathrm{G}_{1} p=0.0017$ ). e Frequency (\%) of apoptotic cells defined by Annexin $\mathrm{V}^{+}$in $\mathrm{CM}$ AML cells after SCR (black) or miRisten (pink/red; $p<0.0001$ for all doses) treatment. f Schematic of treatment regimen. CM AML cells $\left(1 \times 10^{6}\right.$ cells/mouse) were transplanted into WT mice to generate a cohort of AML-bearing mice, which were randomly divided into two groups and treated with SCR control $(20 \mathrm{mg} / \mathrm{kg} /$ dose, i.v., daily; $n=7)$ or miRisten $(20 \mathrm{mg} / \mathrm{kg} /$ dose, i.v., daily; $n=7)$ for 21 days. $\mathbf{g}$ The frequency of uptake for miRisten (CpG-anti-miR-126cy3; red) or vehicle (gray) in c-Kit + AML blasts in BM or PB $1 \mathrm{~h}$ after intravenous (i.v.) treatment with miRisten $20 \mathrm{mg} / \mathrm{kg}$. $\mathbf{h}$ Kaplan-Meier survival curve of CM leukemic mice treated with SCR control (black line; $n=7$; median survival 78 days) or miRisten (red line; $n=7$; median survival 154 days). Dotted line with gray shade indicates treatment window; statistical difference for survival curves was determined using Log-rank (Mantel-Cox) test $(p=0.0013)$. Each dot in $\mathbf{c}, \mathbf{d}$, e represents result from an individual mouse and data are presented as the mean \pm SEM; statistics were calculated using two-tailed Student's $T$ tests $\left({ }^{\star} p<0.05 ;{ }^{\star \star} p<0.01 ;{ }^{\star \star \star} p<0.001\right)$.

Of note, we show that miR-126 functions to enhance MYC protein stability and activity via downregulating SPRED1 and PLK2, negative regulators of RAS-MEK-ERK pathway which in turn regulates $\mathrm{p}$-MYC (S62). Pulikkan et al. ${ }^{59}$ previously demonstrated that $\mathrm{CM}$ maintains MYC expression by relieving RUNX1-mediated repression and that MYC expression is critical for the survival of inv(16) leukemia-initiating cells and AML cells. Our results reveal an additional miR-126-mediated regulatory axis converging on MYC, a critical pathway for CM-driven AML. Given the functional divergence of miR-126, the effects of miR-126on regulation of genes and pathways may be highly dependent on the specific miR-126 level within specific cellular compartment and/or oncogenic context. Our results confirmed that deletion of miR-126 did not result in detectable difference in the immunophenotype or frequencies of normal HSPC subsets (Supplementary Fig. 3a-d). Notably, Lechman et al. reported that 
a

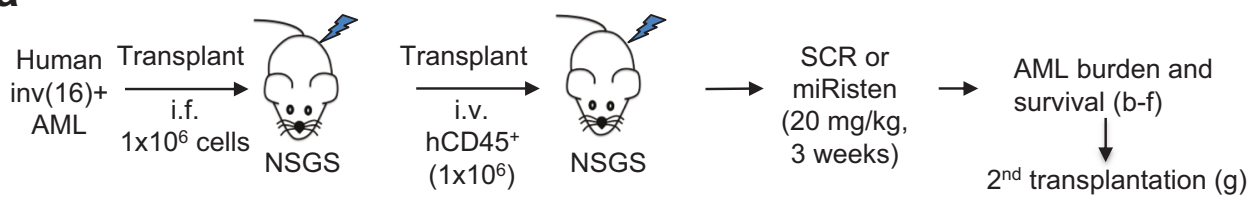

b

BM

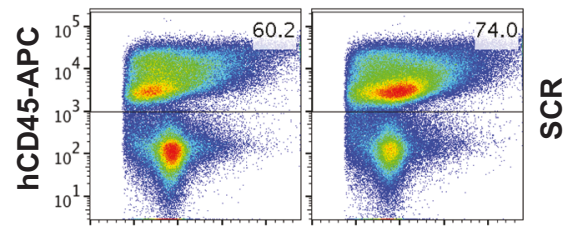

C

BM
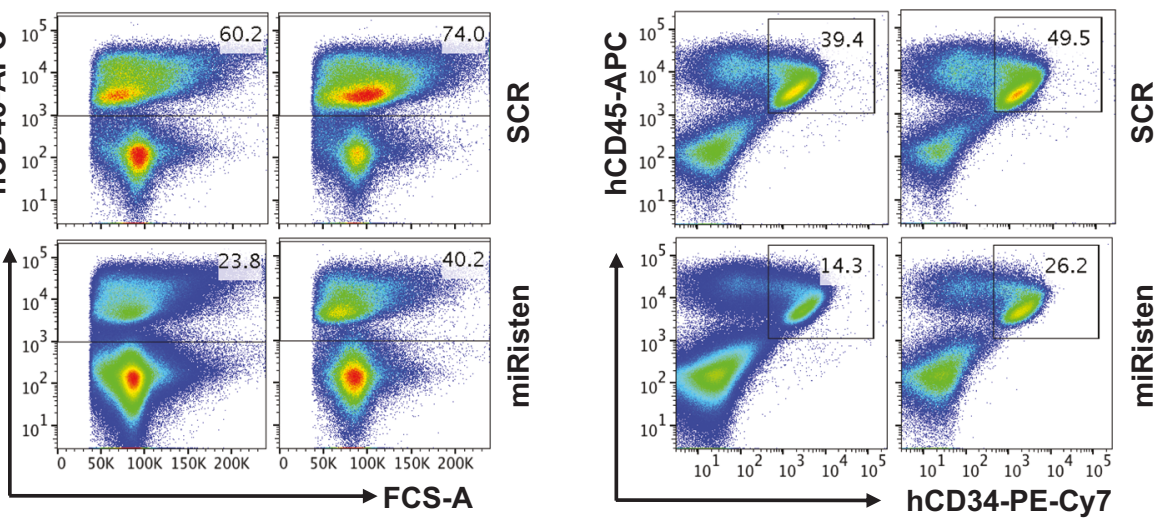

d

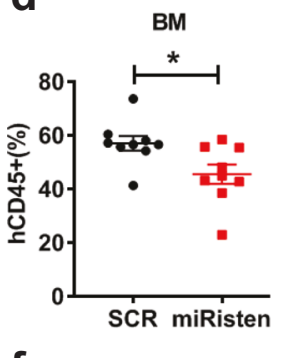

SP

e
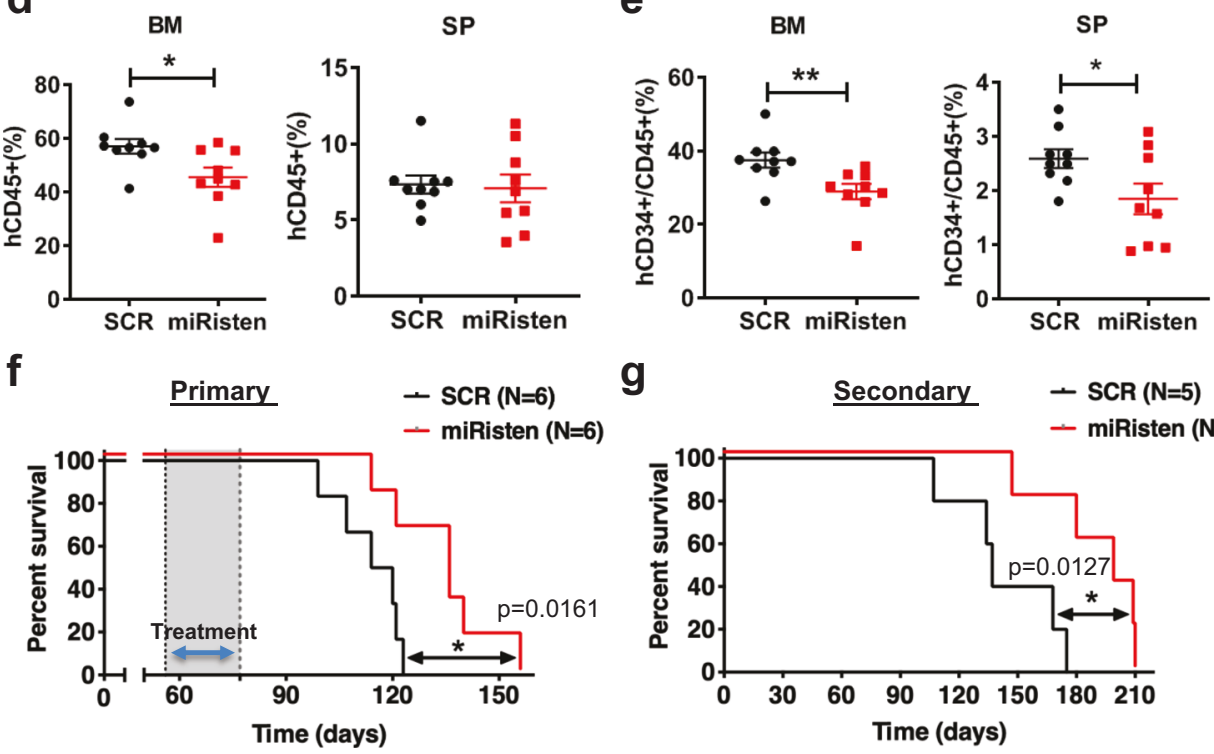

g
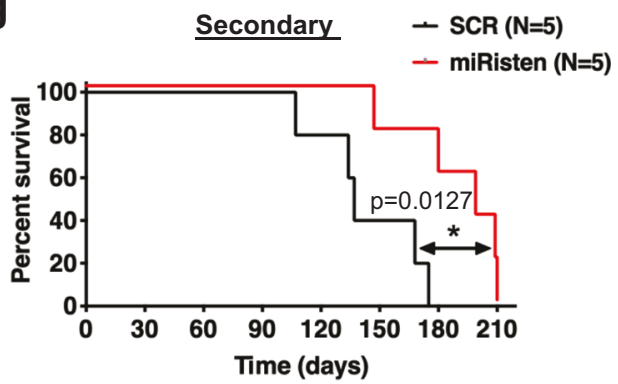

Fig. 7 Administration of miRisten effectively inhibits AML propagation and leukemia-initiating activity in inv(16) AML PDX model. a Schematic of experimental design. Inv(16) AML PDX was established by directly injecting T cell-depleted primary AML cells $\left(1 \times 10^{6}\right.$ cells $/$ mouse) i.f. into irradiated NSGS and subsequently expanded into larger cohorts by secondary transplantation via i.v. injection. After 8 weeks, PDX mice were treated with SCR control ( $20 \mathrm{mg} / \mathrm{kg} /$ dose, i.v., daily) or miRisten $(20 \mathrm{mg} / \mathrm{kg} /$ dose, i.v., daily) for 3 weeks and followed by assessment of human cell engraftment in BM and spleen. b Representative FACS plots showing gating strategy and frequency of hCD45+ cells in BM of mice treated with SCR control (top) or miRisten (bottom) for 3 weeks. c Representative FACS plots showing gating and frequency of hCD34 ${ }^{+} / \mathrm{CD} 45^{+}$cells in BM of mice treated with SCR (top) or miRisten (bottom) for 3 weeks. d Frequency of hCD45+ AML cells in SCR (black) vs. miRisten (red) treated mice $(n=9 / \mathrm{group}) \mathrm{BM}(p=0.0222)$ or spleen (SP). e Frequency of hCD34+/CD45+ AML cells in SCR (black) vs. miRisten (red) treated mice $(n=9 /$ group) BM $(p=0.01)$ or SP $(p=0.0385)$. $\mathbf{f}$ The Kaplan-Meier survival curve of mice treated with SCR (black line; $n=6$; median survival 117 days) or miRisten (red line; $n=6$; median survival 136 days; $p=0.0161$ ) for 3 weeks starting at 8 weeks after transplant. Dotted line with gray shade indicates treatment window. $\mathbf{g}$ The Kaplan-Meier survival curve of second transplant recipients treated with SCR (black line; $n=5$; median survival 137 days) or miRisten (red line; $n=5$; median survival 199 days; $p=0.0127$ ). The statistical significance for $\mathbf{f}, \mathbf{g}$ was determined using log-rank (Mantel-Cox) test. Each dot in $\mathbf{d}$, e represents result from an individual mouse and data are presented as the mean $\pm \mathrm{SEM}$; statistical significance was determined using two-tailed Student's $T$-tests $\left({ }^{\star} p<0.05\right.$; ${ }^{\star \star} p<0.01 ;{ }^{\star \star \star} p<0.001$ ).

miR-126 inhibition resulted in LSC exhaustion while promoting normal HSC expansion ${ }^{22,29}$. This divergent self-renewal outcome underscores the opportunity to selectively target LSCs by therapeutically targeting miR-126.
Importantly, we developed a miR-126 inhibitor, miRisten, which was efficiently taken up and effectively reduced miR-126 expression in AML blasts and stem/progenitor cells. We show the on-target anti-leukemia and anti-LSC efficacy of miRisten in 


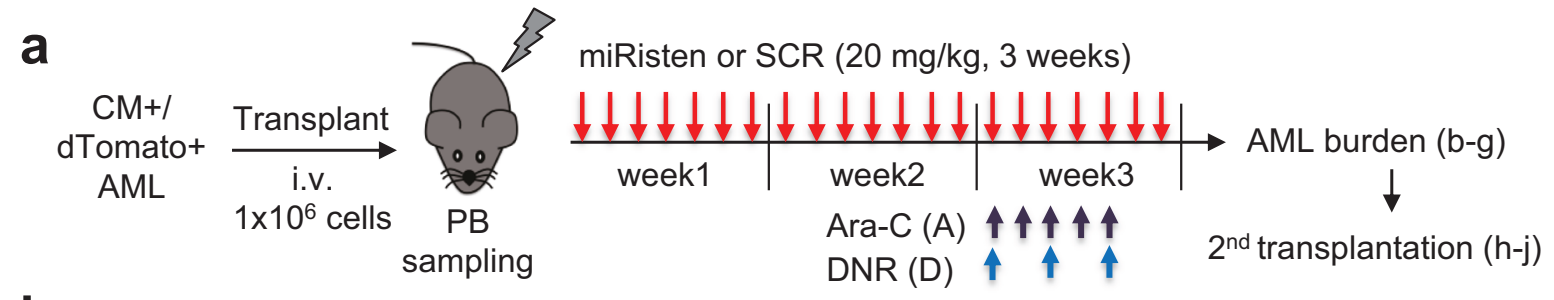

b miRsten - + - + Ara-C - -++ DNR - - + +

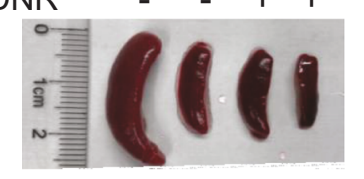

C

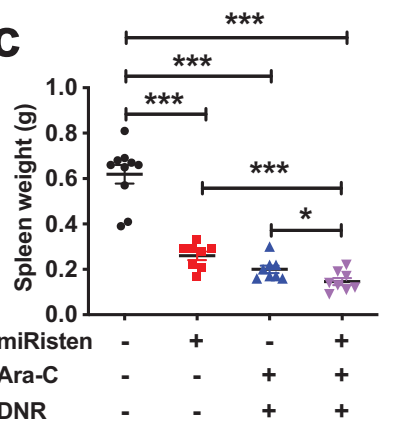

e

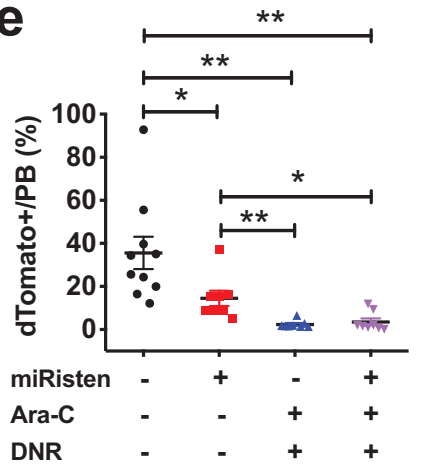

h

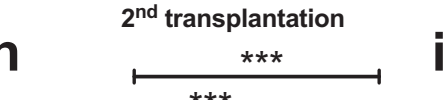

f
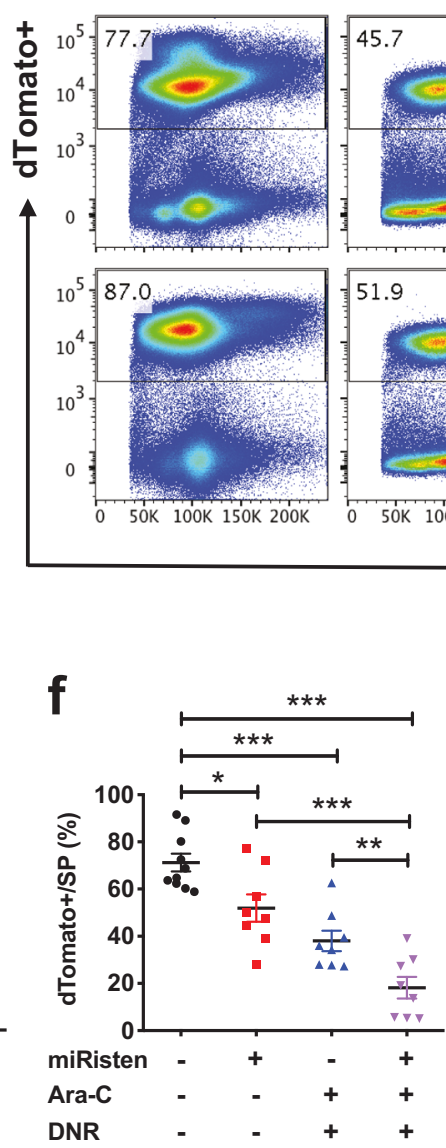

miRisten
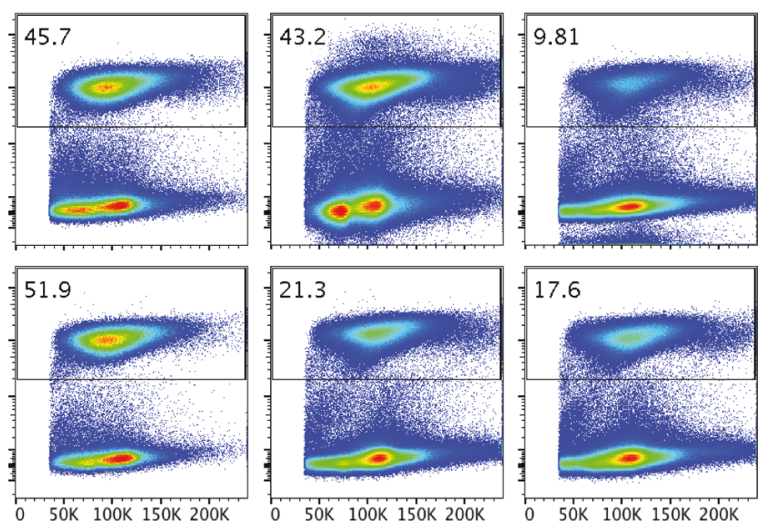

FSC-A

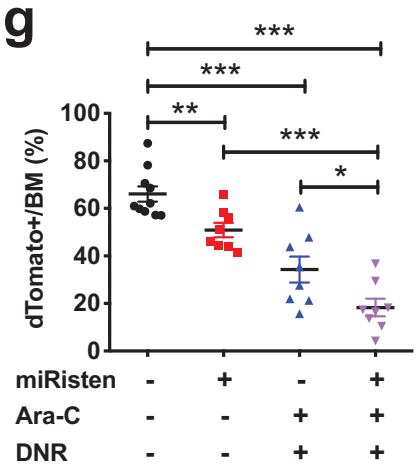

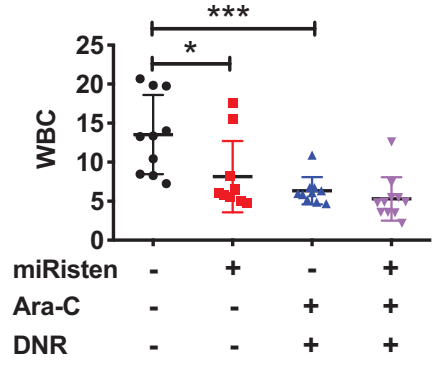
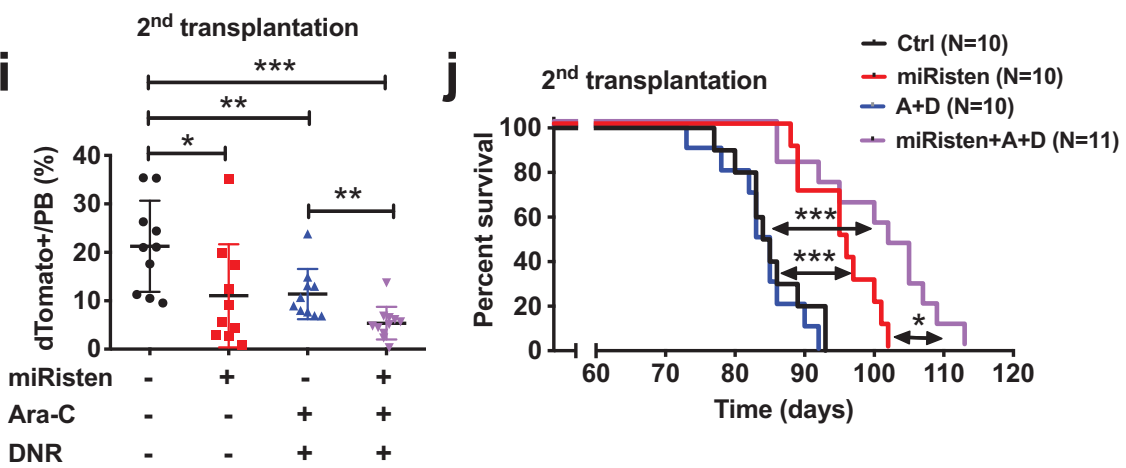

preclinical models of inv(16) AML. In addition, miRisten exhibited similar activities against $\mathrm{t}(8 ; 21)$ AML stem/progenitor cells. Furthermore, we demonstrate that the combination of miRisten with chemotherapy offered improved efficacy in inhibiting leukemia progression and eliminating LSCs. Concordant with our findings, previous studies showed that inhibiting miR126 increased the sensitivity to anti-proliferative drugs or standard chemotherapy in cytogenetically normal $\mathrm{AML}^{29}$ and $t(8,21)$ $\mathrm{AML}^{60}$. This study highlights therapeutically targeting miR-126 as a therapeutic approach for AML patients with inv (16). 
Fig. 8 Anti-miR-126 therapy by miRisten effectively inhibits CM-AML burden and abrogates LSC activity. a Schematic of experimental design. CM/ tdTomato ${ }^{+}$AML cells $\left(1 \times 10^{6}\right)$ were transplanted into cohorts of WTwild-type syngenic mice. After 8 weeks, mice were treated with SCR control or miRisten ( $20 \mathrm{mg} / \mathrm{kg} / \mathrm{dose}$, daily for 3 weeks), or a " $5+3$ " chemotherapy regimen consisted of Ara-C ( $50 \mathrm{mg} / \mathrm{kg} / \mathrm{day}, 5 \mathrm{days}) \mathrm{plus}$ DNR ( $1.5 \mathrm{mg} / \mathrm{kg} / \mathrm{day}$, 3 days), or miRisten and " $5+3$ " chemotherapy combined. AML engraftment was analyzed 7 days after the last dose of treatment and BM cells were transplanted into second recipients, which were analyzed for engraftment at 7 weeks or monitored for leukemia onset and survival. $\mathbf{b}$ Representative images of spleens from recipients of SCR control (Ctrl), miRisten, A+D, or miRisten/A+D group. c Spleen weight from treated recipients of SCR Ctrl (black; $n=10$ ), miRisten (red; $n=8$; miRisten vs. Ctrl $p<0.0001$ ), $A+D$ (blue; $n=8 ; A+D$ vs. Ctrl $p<0.0001$ ), or miRisten/A + D (purple; $n=8$; miRisten/A + D vs. Ctrl $p<0.0001 ;$ miRisten/A + D vs. miRisten $p=0.0004$; miRisten/A+D vs. A + D $p=0.0337)$. d Representative FACS plots showing gating strategy and frequency of dTomato ${ }^{+} \mathrm{AML}$ cells in the BM of SCR control, miRisten, $A+D$, or miRisten/A+D group. e Frequency of dTomato ${ }^{+}$AML cells in the PB of mice treated with SCR control (black; $n=10$ ), miRisten (red; $n=8$; miRisten vs. Ctrl $\left.p=0.0324\right), A+D(b l u e ; n=8$; $\mathrm{A}+\mathrm{D}$ vs. $\mathrm{Ctrl} p=0.0012 ; \mathrm{A}+\mathrm{D}$ vs. miRisten $p=0.0047$ ), or miRisten $\mathrm{A}+\mathrm{D}$ (purple; $n=8$; miRisten/A $+\mathrm{D}$ vs. Ctrl $p=0.0018 ;$ miRisten/A $+\mathrm{D}$ vs. miRisten $p=0.014$ ). $\mathbf{f}$ Frequency of dTomato ${ }^{+}$AML cells in the SP of mice treated with SCR control (black; $n=10$ ), miRisten (red; $n=8 ;$ miRisten vs. Ctrl $p=0.0103$ ),$A+D$ (blue; $n=8 ; A+D$ vs. Ctrl $p<0.0001$ ), or miRisten/A $+\mathrm{D}$ (purple; $n=8 ;$ miRisten/A $+\mathrm{D}$ vs. Ctrl $p<0.0001 ;$ miRisten/A $+\mathrm{D}$ vs. miRisten $p=0.0004$; miRisten $/ A+D$ vs. $A+D p=0.0071$ ). $\mathbf{g}$ Frequency of dTomato ${ }^{+} \mathrm{AML}$ cells in the BM of mice treated with $\mathrm{SCR}$ control (black; $n=10$ ), miRisten (red; $n=8$; miRisten vs. Ctrl $p=0.0038$ ), $A+D$ (blue; $n=8 ; A+D$ vs. Ctrl $p<0.0001$ ), or miRisten/A+D (purple; $n=8 ;$ miRisten/ $A+D$ vs. Ctrl $p<0.0001$; miRisten/A+D vs. miRisten $p<0.0001$; miRisten/A + D vs. $A+D p=0.0291$ ). $\mathbf{h}$ White blood cell (WBC) counts of second transplant recipients of SCR control (black; $n=10$ ), miRisten (red; $n=10 ;$ miRisten vs. Ctrl $p=0.0225), A+D(b l u e ; n=10 ; A+D$ vs. Ctrl $p=0.0005)$, or miRisten/A + D (purple; $n=11$; miRisten/A + D vs. Ctrl $p=0.0002)$ treated mice at 7 weeks. $\mathbf{i}$ Frequency of $\mathrm{dTomato}^{+} \mathrm{AML}$ cells in the PB of second transplant recipients treated with SCR Ctrl (black; $n=10$ ), miRisten (red; $n=10$; miRisten vs. Ctrl $p=0.0358$ ), A + D (blue; $n=10 ; A+D$ vs. Ctrl $p=0.0098$ ), or miRisten/A + D (purple; $n=11 ;$ miRisten/A + D vs. $C \operatorname{trl} p<0.0001 ;$ miRisten/A + D vs. A + D $p=0.0049)$ at 7 weeks. $\mathbf{j}$ The Kaplan-Meier survival curve of second transplant recipients treated with SCR Ctrl (black line; $n=10$; median survival 84.5 days), miRisten (red line; $n=10$; median survival 95.5 days; miRisten vs. Ctrl $p=0.0004$ ), $A+D$ (blue line; $n=10$; median survival 84 days), or miRisten/A + D (purple line; $n=11 ;$ median survival 102 days; miRisten/A + D vs. Ctrl $p=0.0002$; miRisten/A + D vs. miRisten $p=0.0386$ ). The statistical significance was determined using logrank (Mantel-Cox) test. Each dot in $\mathbf{c}, \mathbf{e}, \mathbf{f}, \mathbf{g}, \mathbf{h}$, i represents data from an individual mouse and presented as the mean \pm SEM; statistics for all comparisons shown were determined using two-tailed $T$-tests $\left({ }^{\star} p<0.05 ;{ }^{\star \star} p<0.01 ;{ }^{\star \star \star} p<0.001\right)$.

\begin{abstract}
Methods
Human samples. All AML samples used (see Supplementary Table 3) were from patients who were treated at City of Hope and normal bone marrow or mobilized peripheral blood stem cells were obtained from healthy (HL) donors. Samples were acquired with signed informed consent and the procedure was approved by the City of Hope Institutional Review Board, in accordance with an assurance filed with and approved by the Department of Health and Human Services.
\end{abstract}

Isolation of mononuclear cells from patient samples. The specimen was transferred to a $50 \mathrm{~mL}$ conical tube and the volume was brought up to $25 \mathrm{~mL}$ using warm Dulbecco's phosphate-buffered saline phosphate-buffered saline (DPBS) with $2 \%$ fetal bovine serum (FBS). The specimen was layered on the top of $20 \mathrm{~mL}$ Ficoll-Paque Plus in a $50 \mathrm{~mL}$ conical tube. Then, the tube was centrifuged at $300 \mathrm{~g}$ for $30 \mathrm{~min}$ without break. The layer containing mononuclear cells and plasma was carefully transferred to a $50 \mathrm{~mL}$ conical tube and the volume was brought up to $50 \mathrm{~mL}$ with warm DPBS. The tube was then centrifuge at $1341 \mathrm{~g}$ for $8 \mathrm{~min}$. After discard the supernatant, the pellet was resuspended in $10 \mathrm{~mL}$ of warm DPBS. The cell number and viability were assessed and CD34 ${ }^{+}$ cells were enriched using StemSep ${ }^{\mathrm{TN}}$ Human CD34 Positive Selection reagents (Stemcell Technologies, 14756).

Mice. $C b f b^{56 M /+32,33}$ (available upon request), $m i R 126^{\text {flox/flox34 }}$ (provided by Dr. Calvin J. Kuo, Standford University), and Mx1-Cre ${ }^{67}$ (Jackson Laboratory, Stock No. 003556) mice were backcrossed to C57BL/6 for more than ten generations. Ai14 Cre tdTomato reporter mice (Stock No. 007914) and C57BL/6J mice (Stock No. 000664) were purchased from the Jackson Laboratory. To induce miR-126 deletion and/or CM expression, $C b f b^{56 M /+} / m i R 126^{\text {flox/flox }} / \mathrm{Mx} 1$-Cre mice and $C b f b^{56 M /+} / M x 1-C r e$ (6-8-week-old, both females and males) were injected intraperitoneally (i.p.) with polyinosinic-polycytidylic acid [poly (I:C)] (InvivoGen, tlrlpicw-250) at $14 \mathrm{mg} / \mathrm{kg} /$ dose every other day for a total of seven doses. Age-matched $C b f b^{56 M /+}$, miR126flox/flox, or Cbfb $56 M /+/ m i R 126^{\text {flox/flox }}$ littermates were similarly poly (I:C) treated and used as controls. Preleukemic analysis was performed 6 weeks after the last dose of poly (I:C) injection. Mice were monitored for leukemia development and survival for up to 1 year. AML transplant was performed via tail vein i.v. injection into sublethally irradiated (X-RAD 320-Precision X-Ray; 4.5 Gy) 6-8 week-old syngenic C57BL/6 wild-type (WT) mice. In vivo administration of miRisten or scramble (SCR) control was performed by daily retro-orbital i.v. injection. Mice were maintained on $12 \mathrm{~h}$ light $/ 12 \mathrm{~h}$ dark cycles under $18-23^{\circ} \mathrm{C}$ ambient temperature with 40-60\% humidity in an Association for Assessment and Accreditation of Laboratory Animal Care-accredited animal facility. All experimental procedures were performed in accordance with federal and state government guidelines and established institutional guidelines and protocols approved by the Institutional Animal Care and Use Committee at City of Hope.

Cell isolation and flow cytometry. BM mononuclear cells were collected from femurs, tibias, and pelvis using a mortar and pestle $33,36,37$. For fluorescence activated cell-sorting (FACS) analyses, cells were stained with fluorescently labeled antibodies in phosphate-buffered saline (PBS) with $0.5 \%$ bovine serum albumin (BSA) for $15 \mathrm{~min}$ at $4{ }^{\circ} \mathrm{C}$. Antibodies used included are listed in Supplementary Table 4 . Flow cytometry was performed using a 5-laser, BD LSRFortessa ${ }^{\mathrm{Tx}} \mathrm{X}-20$ cell analyzer. For cell sorting, lineage-negative $\left(\mathrm{Lin}^{-}\right)$cells were first enriched using EasySep ${ }^{\text {th }}$ negative selection reagents (Stemcell Technologies, 18000) and then sorted on a 5-Laser, BD FACSAria Fusion Cell Sorter. Acquired data were analyzed by Flowjo software 10.6.1. Phenotypic HSPC populations included long-term HSCs (LT-HSC; $\mathrm{Lin}^{-} \mathrm{cKit}^{+} \mathrm{Scal}{ }^{+} \mathrm{CD} 48^{-}$ CD150 ${ }^{+}$), short-term HSCs (ST-HSC; $\mathrm{Lin}^{-} \mathrm{cKit}^{+} \mathrm{Scal}^{+} \mathrm{CD}^{-} 8^{-} \mathrm{CD} 150^{-}$), multipotent progenitors (MPP; $\mathrm{Lin}^{-} \mathrm{cKit}^{+} \mathrm{Sca}{ }^{+} \mathrm{CD} 48^{+} \mathrm{CD} 150^{+/-}$), LSK $\left(\mathrm{Lin}^{-} \mathrm{cKit}^{+} \mathrm{Scal}^{+}\right.$), pregranulocyte-macrophage (Pre-GM; $\mathrm{Lin}^{-} \mathrm{ckit}^{+} \mathrm{Scal}{ }^{-} \mathrm{CD} 16 / 32^{-/ \mathrm{lo}} \mathrm{CD} 105^{-} \mathrm{CD} 150^{-}$), granulocyte-macrophage progenitors (GMP; $\mathrm{Lin}^{-} \mathrm{ckit}^{+} \mathrm{Sca}{ }^{-} \mathrm{CD} 16 / 32^{+} \mathrm{CD} 150^{-}$), pre-megakaryocyte/erythrocyte (Pre-Meg/E; $\mathrm{Lin}^{-} \mathrm{ckit}^{+} \mathrm{Sca}{ }^{-} \mathrm{CD} 16 / 32^{-/ \mathrm{lo}} \mathrm{CD} 105^{-}$ $\mathrm{CD}_{150^{+}}$), and erythroid progenitors (EP; $\mathrm{Lin}^{-} \mathrm{ckit}^{+} \mathrm{Scal}{ }^{-} \mathrm{CD} 16 / 32^{-/ \mathrm{lo}^{\circ}} \mathrm{CD} 105^{+}$, including Pre-CFU-E, CFU-E, Pro-erythrocytes) ${ }^{33,36,37 .}$

RNA isolation and reverse transcription. Total RNA, including miRNA, was isolated using AllPrep DNA/RNA/miRNA Universal Kit (Qiagen, 80224) following the manufacturer's protocol. First-strand cDNA was generated using SuperScript ${ }^{\mathrm{m}}$ IV Reverse Transcriptase (Thermo Fisher Scientific, 18090010). Reverse transcription was performed using Applied Biosystems ${ }^{\circledR}$ TaqMan $^{\circledR}$ MicroRNA Reverse Transcription Kit (Thermo Fisher Scientific, 4366596) and the gene specific primers [miR-126 (002228), Sno234 (001234), and RNU44 (001094)] provided by TaqMan ${ }^{\circledR}$ MicroRNA Assay.

Quantitative PCR. Quantitative PCR was performed using TaqMan ${ }^{\text {Th }}$ Universal Master Mix II, with UNG (Thermo Fisher Scientific, 4440038) or SYBR ${ }^{\mathrm{Tw}}$ Green PCR Master Mix (Thermo Fisher Scientific, 4368577). TaqMan Assays and primer sequences are listed in Supplementary Tables 5 and 6. Results were normalized to internal reference genes by the $\mathrm{Ct}$ method, and relative expression was calculated as $2^{-\Delta \mathrm{Ct}}$.

Design and synthesis of miRisten (CpG-anti-miR-126). The partially phosphothioated anti-miR-126 or scrambled (SCR) RNA was linked using 5 U of C3 carbon chain linker, $\left(\mathrm{CH}_{2}\right)_{3}$ (indicated by " $\mathrm{x}$ "). The constructs were also conjugated with $\mathrm{Cy} 3$ to track the internalization in cells by flow cytometry. The sequences were as follows: CpG-anti-miR-126: 5'-G*G*TGCATCGATGCAGG*G* ${ }^{*}{ }^{*} G^{*}$ Gxxxxx mCmGmCmAmUmUmAmUmUmAmC mUmCmAmCmGmGmUmAmCmGm A-3'; CpG-SCR: 5'-G* G $^{*}$ TGCATCGATGCAGG* $\mathrm{G}^{*} \mathrm{G}^{*} \mathrm{G}^{*} \mathrm{G}$ xxxxxmGmUmA mGmAmAmCmCmGmUmAmCmUmCmGmUmCmAmCmUmUmA-3', where the asterisk $\left(^{*}\right)$ indicates phosphorothioation. One nonbridging atom of oxygen on phosphate was replaced with sulfur. " $\mathrm{m}$ " indicates the $2^{\prime}$-O-methyl analog of the nucleotide. 
Xenograft mouse model. Human inv(16) AML cells were transplanted via i.f. injection into sublethally irradiated (2 Gy) 6-8-week-old NOD/SCID/IL-2R- $\gamma^{-/-/}$ Tg (CMV-IL3, CSF2, KITLG) mice (NSGS) purchased from the Jackson Laboratory (Stock No. 013062). Leukemia BM cells were collected and transplanted $\left(1 \times 10^{6}\right.$ cells $)$ via tail vein i.v. injection into a larger cohort of NSGS mice. Engraftment of human (h)CD45 cells in PB was monitored by flow cytometry at 6-8 weeks after transplantation. Mice were treated with miRisten or SCR control by retro-orbital i.v. injection $(20 \mathrm{mg} / \mathrm{kg} / \mathrm{dose})$ daily for 21 days. This dose and schedule were selected based on empirical testing showing effective knockdown, efficacy, tolerability, and feasibility of drug synthesis. After treatment, PB and BM cells were collected and assessed for AML burden by flow cytometry (see antibodies in Supplementary Table 4).

Virus production and transduction of primary AML and 32D cells. The miRZip anti-miR-126-3p-GFP co-expressing plasmid (CS940MZ-1, a custom order from System Biosciences, with EEF1A1 promoter for anti-miR-126-3p) and control plasmid (MZIP000-PA-1, miRZip negative control) were purchased from System Biosciences. Virus were produced and transduced as previously described ${ }^{30}$. Briefly, primary AML or enriched CD34+ cells were cultured in Gibco IMDM (Thermo Fisher Scientific, 12440061) supplemented with 20\% FBS and growth factors including interleukin 3 (IL-3, $25 \mathrm{ng} / \mathrm{mL}$ ), interleukin 6 (IL-6, $10 \mathrm{ng} / \mathrm{mL}$ ), Fms-like tyrosine kinase 3 ligand (Flt-3 ligand, $100 \mathrm{ng} / \mathrm{mL}$ ), stem cell factor (SCF, $50 \mathrm{ng} / \mathrm{mL}$ ), and thrombopoietin $(100 \mathrm{ng} / \mathrm{mL})$ overnight before transduction. Transduction was performed with a multiplicity of infection (MOI) of 25 by spinoculation in the presence of $1 \times$ TransDux MAX ${ }^{\mathrm{mi}}$ (System Biosciences, LV860A-1). 32D cells were cultured in RPMI-1640 supplemented with 10\% FBS, 20\% WEHI-3 conditioned media, and antibiotics at $37^{\circ} \mathrm{C}$ with $5 \% \mathrm{CO}_{2}$ and were transduced with MSCVIRES-GFP (MIG)-based retroviruses or lentiviruses (CD530, pLKO.1 or HIV-7) $(\mathrm{MOI}=10)$ by spinoculation in the presence of $8 \mathrm{ug} / \mathrm{mL}$ polybrene (American Bioanalytical, AB01643-00001). Two days after infection, $\mathrm{GFP}^{+}$or $\mathrm{RFP}^{+}$cells was sorted on a 5-Laser, BD FACSAria Fusion Cell Sorter. The target sequences for shRNAs are shown in Supplementary Table 7.

Transfection of 32D cells with synthetic small interfering RNA (siRNA) oligonucleotides. 32D cells were transfected by siEbpl (the siGENOME SMARTpool, J-00860-05), which purchased from Thermo Fisher Scientific and scrambled control RNA (siCtrl) using electroporation with SF Cell Line 4D-Nucleofector ${ }^{\mathrm{TM}} \mathrm{X}$ Kit (Lonza, V4XC-2032) via program CV-137 following the manufacturer's protocol. The target sequences for siRNAs are shown in Supplementary Table 7.

Chromatin immunoprecipitation. Cells are fixed with formaldehyde and lysed according to the SimpleChIP ${ }^{\oplus}$ Plus Enzymatic Chromatin IP Kit (Magnetic Beads, Cell Signaling Technology, 9005). After chromatin is digested, immunoprecipitations are performed using ChIP-validated antibodies: Anti-FLAG (Cell Signaling Technology, 14793), CBFb Antibody-ChIP-seq Grade (Diagenode, C15310002), Recombinant Anti-RUNX1/AML1 antibody (Abcam, ab272456), Anti-GATA2 antibody-ChIP Grade (Abcam, ab22849), and ChIP-Grade Protein G Magnetic Beads. After reversal of protein-DNA cross-links, the DNA is purified using DNA purification spin columns, following with quantitative real-time PCR. Fold enrichment was calculated with equation: $2^{-(\mathrm{C}[\mathrm{T}]}$ IP Sample - C[T] IP IgG).

Immunocytochemistry. Cells were collected, washed in ice-cold PBS and mounted on glass slides using a Cytocentrifuge (CytoSpin4, 91g, $10 \mathrm{~min}$ ). Cells were then washed with PBS, fixed in $4 \%$ paraformaldehyde for $15 \mathrm{~min}$ and permeabilized in $0.5 \%$ Triton X-100 for $15 \mathrm{~min}$. Non-specific epitopes were blocked with 5\% BSA for $30 \mathrm{~min}$. Primary antibodies used are listed in Supplementary Table 8. Secondary anti-mouse/rabbit/goat-Alexa 594/488/647 antibodies were purchased from Thermo Fisher Scientific. Cell images were acquired using a Zeiss confocal laserscanning-microscope (Zeiss LSM 800). Nuclei were counterstained with ProLong $^{\mathrm{TM}}$ Gold Antifade Mountant with DAPI (Thermo Fisher Scientific, P36931).

Western blotting and immunoprecipitation. Cells were washed and harvested in ice-cold PBS and subsequently lysed in radioimmunoprecipitation assay (RIPA) buffer containing $10 \mathrm{mM}$ protease inhibitor cocktail (Thermo Fisher Scientific, 78429). For immunoprecipitation, $500 \mu \mathrm{g}$ to $1 \mathrm{mg}$ of cell lysates were incubated with indicated antibody for overnight at $4{ }^{\circ} \mathrm{C}$. Fifty microliters of Protein $\mathrm{A} / \mathrm{G}$ agarose beads (Cell Signaling Technology, 9863; 37478) were added, and then incubated at $4{ }^{\circ} \mathrm{C}$ for $3 \mathrm{~h}$. For immunoblotting, immunoprecipitated complex or $50 \mu \mathrm{g}$ of each cell lysate was separated on NuPAGE $4-12 \%$ gradient gels (Thermo Fisher Scientific), and immunocomplexes were visualized with enhanced chemiluminescence reagent (Thermo Fisher Scientific, 34095). The information for antibodies used is presented in Supplementary Table 8.

Ubiquitination assay. Cells were washed twice with PBS and then $50 \mu \mathrm{L}$ of lysis buffer (Tris-buffered saline [TBS] and $2 \%$ sodium dodecyl sulfate [SDS]) was added and boiled. Two hundred and fifty microliters TBS supplemented with $1 \%$ Triton X-100 was added and the mixture was sonicated. After centrifugation, the supernatant was immunoprecipitated using anti-HDAC8 antibody following capture by protein G plus protein A agarose mixture (Calbiochem ${ }^{\oplus}$, IP05). The bound proteins were eluted using $4 \times$ SDS sample buffer boiling at $100^{\circ} \mathrm{C}$ for $5 \mathrm{~min}$, resolved on NuPAGE 4-12\% gradient gels (Thermo Fisher Scientific), and detected by anti-ubiquitin antibody.

RAN activation assay. RAN activation assay (Cell Biolabs, STA-409) was performed to measure activities of RAN according to the manufacturer's guidance. Briefly, the cells were washed twice with ice-cold PBS and lysed in lysis buffer with passing through a $271 / 2$-gauge serine to shear the genomic DNA. The lysate was centrifuged, and supernatant was collected. RanBP1 PBD agarose bead was added and the complex was incubated at $4{ }^{\circ} \mathrm{C}$ for $1 \mathrm{~h}$ with gentle agitation. For positive and negative controls, GTP $\gamma \mathrm{S}$ and GDP were added, respectively. Then the beads were collected, resuspended with $2 \times$ SDS-PAGE sample buffers, and boiled for 5 min. Twenty microliters of pulldown sample was loaded to NuPAGE $4-12 \%$ gradient gels (Thermo Fisher Scientific) and detected using anti-RAN antibody.

Luciferase reporter assay. $293 \mathrm{~T}$ cells were co-transfected with pGL3BasicmiR126-EGFL7-Promoter plasmid (Addgene, 32244) derived from pGL3.0 vector and Renilla luciferase reporter vector (phRL-TK) combined with pMIG-CM or pMIG-CBFb and pMIG-RUNX1 or empty control vector. After transfection ( $48 \mathrm{~h}$ ), the activities of firefly and Renilla luciferases were measured sequentially from a single sample using the Dual-Luciferase ${ }^{\circledast}$ Reporter Assay System (Promega, E1910) according to the manufacturer's protocol. Briefly, cultured cells were rinsed with $1 \times$ PBS and lysed by $1 \times$ Passive Lysis Buffer (PLB) solution (Promega, E1941). Homogeneous lysates were prepared by manually scraping the cells from culture wells in the presence of $1 \times$ PLB and then transferred into a tube. The firefly luciferase reporter is measured first by adding Luciferase Assay Reagent II (LAR II) to generate a stabilized luminescent signal. After quantifying the firefly luminescence, this reaction is quenched, and the Renilla luciferase reaction is simultaneously initiated by adding Stop \& $\mathrm{Glo}^{\circledast}$ Reagent to the same tube and then measured Renilla luminescence. Measurements were read using a white, flat bottom 96-well plate (Costar, 3912) on a GloMax ${ }^{\circledast} 20 / 20$ Luminometer. The normalized luciferase ratio is obtained for each well by calculating "firefly reporter activity/Renilla reporter activity".

Small RNA library preparation and next-generation sequencing (NGS). All libraries were prepared using the Illumina TruSeq Small RNA protocol with minor modification following the manufacturer's instructions. Briefly, $280 \mathrm{ng}$ of pooled total RNA was ligated to the sRNA $3^{\prime}$ adaptor (TCT GGA ATT CTC GGG TGC CAA GGA ACT CC) with T4 RNA Ligase 2, truncated (New England BioLabs) for $1 \mathrm{~h}$ at $22^{\circ} \mathrm{C}$, and subsequently ligated to a $5^{\prime}$ adaptor $(0.5 \mu \mathrm{L}$ of $5 \mu \mathrm{M}$ per reaction): GUUCAGAGUUCUACAGUCCGACGAUCNNN with T4 RNA ligasel (New England BioLabs) for $1 \mathrm{~h}$ at $20^{\circ} \mathrm{C}$. The constructed smRNA library was first reverse-transcribed using GX1 (5'-GGAGTTCCTTGGCACCCGAGA) as the RT primer and then subjected to PCR amplification for 13 cycles, using primers GX1 (5'-CAAGCAGAAGACGGCATACGAGAT[NNNNNN]GTGACTGGAGTTCCTTGGCACCCGAGAATTCCA) and GX2 (5'-AATGATACGGCGACCACCGAGATCTACACTCTT TCCCTACACGACGCT CTTCCGATCT) then followed by 6\% TBE PAGE gel purification with size selection (for targeted small RNAs of 17-35 nt). The purified library was quantified using qPCR with a forward primer (5'-CAAGCAGAAGACGGCATACG) and a reverse primer (5'-AATGATACGGCGACCACCGA). Individual libraries were prepared using a unique index primer in order to allow for pooling of multiple samples prior to sequencing. The library was quantified using qPCR. Sequencing of 50 cycles was performed on a HiSeq 2500 (Illumina Inc., San Diego, CA), and image processing and base calling were conducted using Illumina's pipeline.

miRNA-seq data analysis. Raw small RNA sequences were trimmed to remove the $3^{\prime}$-adapter (TCTGGAATTCTCGGGTGCCAAGGAACTCC) using cutadapt v0.9.3. Reads longer than $16 \mathrm{bp}$ after trimming were aligned to mouse genome assembly $\mathrm{mm} 9$ using Bowtie v 0.12 .7 with default settings. The expression level of mouse mature miRNAs from Sanger mirBase v18 were counted as previously described $^{68}$. The counts of miRNAs were then normalized by the trimmed mean of M-values (TMM) method and counts per million (CPM) values were calculated by Bioconductor package "edgeR" v3.4.2.

RNA-seq and bioinformatics. Total RNA was extracted using the AllPrep DNA/ RNA/miRNA Universal Kit (Qiagen, 80224); quality and quantity were estimated using BioAnalyser Systems (Agilent Technologies). Samples with a RIN $>8.0$ were included. External RNA Controls Consortium (ERCC) Spike-In Control Mix (Thermo Fisher Scientific, 4456740) was added to all samples per the manufacturer's recommendations, although these were not used for downstream analyses. Sequencing libraries were constructed using the KAPA RNA HyperPrep Kit with RiboErase (HMR) (Roche, KK8560), loaded on to a cBot system for cluster generation, and sequenced on a Hiseq 2500 System (Illumina) with single end 51$\mathrm{bp}$ for mRNA-seq to a nominal depth of 50 million reads. Raw RNA-seq sequences were subjected to adapter trimming using Trimmomatic $\mathrm{v} 0.38$ and poly(A) tails were removed using FASTP v0.19.4. The trimmed reads were aligned to mouse genome mm10 using Tophat v2.0.8 with default settings. Expression level of RefSeq 
gene (downloaded on 02/13/2020) were counted using HTSeq count v0.6.1. The raw count data were normalized by the TMM method using Bioconductor package "edgeR" v3.20.9. Differential expression analysis was carried out using the quasilikelihood (QL) F-test implemented in "edgeR" to determine the differentially expressed genes, with the cutoff of average RPKM in one group $\geq 1, p$ values $\leq 0.01$ and fold change $\geq 1.5$. The gene set enrichment analysis $\left(\right.$ GSEA $^{69}$ ) was performed by ClusterProfiler v3.10.1 to identify the affected GO, hallmark and KEGG pathways from $\mathrm{MSigDB} 7^{70}$, using the pre-ranked gene list sorted by the $-\log 10(p$ value) with a sign determined by the fold change direction.

Statistics and reproducibility. Comparison between groups was performed by a two-tailed, paired or unpaired Student's T-test, one-way or two-way ANOVA for normal distributions. The log-rank test was used to assess significant differences between survival curves. All statistical analyses were performed using Prism version 8.0 software (GraphPad Software). Sample sizes chosen are indicated in the individual figure legends and were not based on formal power calculations to detect prespecified effect sizes. A $p$ value less than 0.05 was considered statistically significant. All experiments are repeated independently with similar results at least two times.

Reporting summary. Further information on research design is available in the Nature Research Reporting Summary linked to this article.

\section{Data availability}

The raw data and processed RNA-seq data for LSK cells generated in this study have been deposited in the GEO repository under accession number GSE184015, which can be assessed with no restriction. The raw data and processed RNA-seq data associated with Fig. 1D, S1C was deposited under GEO accession number GSE133642, which can be assessed with no restriction. Raw miRNA-seq data associated with Fig. 1C were deposited to GEO under accession number GSE173785. The uncropped blots generated in this study are provided in the Supplementary Information/Source data file. Source data are provided with this paper.

Received: 29 April 2021; Accepted: 5 October 2021;

Published online: 22 October 2021

\section{References}

1. Bloomfield, C. D., Marcucci, G., Dohner, K. \& Dohner, H. Acute myeloid leukemia. Introduction. Semin. Oncol. 35, 324-325 (2008).

2. Hourigan, C. S. Acute myeloid leukemia: Introduction. Semin. Hematol. 52, 149 (2015).

3. Thomas, D. \& Majeti, R. Biology and relevance of human acute myeloid leukemia stem cells. Blood 129, 1577-1585 (2017).

4. Pollyea, D. A. \& Jordan, C. T. Therapeutic targeting of acute myeloid leukemia stem cells. Blood 129, 1627-1635 (2017).

5. Dohner, H. et al. Diagnosis and management of AML in adults: 2017 ELN recommendations from an international expert panel. Blood 129, 424-447 (2017).

6. Khaled, S., Al Malki, M. \& Marcucci, G. Acute myeloid leukemia: biologic, prognostic, and therapeutic insights. Oncology (Williston Park) 30, 318-329 (2016).

7. Liu, P. P. et al. Identification of the chimeric protein product of the CBFBMYH11 fusion gene in inv(16) leukemia cells. Genes Chromosomes Cancer 16, 77-87 (1996)

8. Mrózek, K., Heerema, N. A. \& Bloomfield, C. D. Cytogenetics in acute leukemia. Blood Rev. 18, 115-136 (2004).

9. de Bruijn, M. F. \& Speck, N. A. Core-binding factors in hematopoiesis and immune function. Oncogene 23, 4238-4248 (2004)

10. Speck, N. A. \& Gilliland, D. G. Core-binding factors in haematopoiesis and leukaemia. Nat. Rev. Cancer 2, 502-513 (2002).

11. Liu, P. et al. Fusion between transcription factor CBF beta/PEBP2 beta and a myosin heavy chain in acute myeloid leukemia. Science 261, 1041-1044 (1993).

12. Prebet, T. et al. Acute myeloid leukemia with translocation $(8 ; 21)$ or inversion (16) in elderly patients treated with conventional chemotherapy: a collaborative study of the French CBF-AML intergroup. J. Clin. Oncol. 27, 4747-4753 (2009).

13. Ustun, C. et al. Core-binding factor acute myeloid leukemia with $\operatorname{inv}(16)$ : older age and high white blood cell count are risk factors for treatment failure. Int. J. Lab. Hematol. 43 e19-e25 (2020).

14. Ambros, V. The functions of animal microRNAs. Nature 431, 350-355 (2004)

15. Bartel, D. P. MicroRNAs: genomics, biogenesis, mechanism, and function. Cell 116, 281-297 (2004).

16. Bohnsack, M. T., Czaplinski, K. \& Gorlich, D. Exportin 5 is a RanGTPdependent dsRNA-binding protein that mediates nuclear export of premiRNAs. RNA 10, 185-191 (2004).

17. Lund, E., Guttinger, S., Calado, A., Dahlberg, J. E. \& Kutay, U. Nuclear export of microRNA precursors. Science 303, 95-98 (2004).
18. Ha, M. \& Kim, V. N. Regulation of microRNA biogenesis. Nat. Rev. Mol. Cell Biol. 15, 509-524 (2014).

19. Chen, C. Z., Li, L., Lodish, H. F. \& Bartel, D. P. MicroRNAs modulate hematopoietic lineage differentiation. Science 303, 83-86 (2004).

20. Wang, S. et al. The endothelial-specific microRNA miR-126 governs vascular integrity and angiogenesis. Dev. Cell 15, 261-271 (2008).

21. Fish, J. E. et al. miR-126 regulates angiogenic signaling and vascular integrity. Dev. Cell 15, 272-284 (2008).

22. Lechman, E. R. et al. Attenuation of miR-126 activity expands HSC in vivo without exhaustion. Cell Stem Cell 11, 799-811 (2012)

23. Li, Z. et al. Distinct microRNA expression profiles in acute myeloid leukemia with common translocations. Proc. Natl Acad. Sci. USA 105, 15535-15540 (2008).

24. Jongen-Lavrencic, M., Sun, S. M., Dijkstra, M. K., Valk, P. J. \& Lowenberg, B. MicroRNA expression profiling in relation to the genetic heterogeneity of acute myeloid leukemia. Blood 111, 5078-5085 (2008).

25. Garzon, R. et al. MicroRNA signatures associated with cytogenetics and prognosis in acute myeloid leukemia. Blood 111, 3183-3189 (2008).

26. Cammarata, G. et al. Differential expression of specific microRNA and their targets in acute myeloid leukemia. Am. J. Hematol. 85, 331-339 (2010).

27. Marcucci, G., Radmacher, M. D., Mrozek, K. \& Bloomfield, C. D. MicroRNA expression in acute myeloid leukemia. Curr. Hematol. Malig. Rep. 4, 83-88 (2009).

28. de Leeuw, D. C. et al. Attenuation of microRNA-126 expression that drives CD34+38- stem/progenitor cells in acute myeloid leukemia leads to tumor eradication. Cancer Res 74, 2094-2105 (2014).

29. Lechman, E. R. et al. miR-126 regulates distinct self-renewal outcomes in normal and malignant hematopoietic stem cells. Cancer Cell 29, 602-606 (2016).

30. Zhang, B. et al. Bone marrow niche trafficking of miR-126 controls the selfrenewal of leukemia stem cells in chronic myelogenous leukemia. Nat. Med. 24, 450-462 (2018).

31. Nguyen, L. X. T. et al. Cytoplasmic DROSHA and non-canonical mechanisms of miR-155 biogenesis in FLT3-ITD acute myeloid leukemia. Leukemia 35, 2285-2298 (2021)

32. Kuo, Y. H. et al. Cbf beta-SMMHC induces distinct abnormal myeloid progenitors able to develop acute myeloid leukemia. Cancer Cell 9, 57-68 (2006)

33. Cai, Q. et al. CBFbeta-SMMHC creates aberrant megakaryocyte-erythroid progenitors prone to leukemia initiation in mice. Blood 128, 1503-1515 (2016).

34. Kuhnert, F. et al. Attribution of vascular phenotypes of the murine Egfl7 locus to the microRNA miR-126. Development 135, 3989-3993 (2008).

35. Parker, L. H. et al. The endothelial-cell-derived secreted factor Egfl7 regulates vascular tube formation. Nature 428, 754-758 (2004).

36. Hua, W. K. et al. HDAC8 regulates long-term hematopoietic stem-cell maintenance under stress by modulating p53 activity. Blood 130, 2619-2630 (2017).

37. Jeannet, R., Cai, Q., Liu, H., Vu, H. \& Kuo, Y.-H. Alcam Regulates long-term hematopoietic stem cell engraftment and self-renewal. Stem Cells 31, 560-571 (2013).

38. Saida, S. et al. Gata2 deficiency delays leukemogenesis while contributing to aggressive leukemia phenotype in Cbfb-MYH11 knockin mice. Leukemia 34, 759-770 (2020)

39. Hartmann, D. et al. MicroRNA-based therapy of GATA2-deficient vascular disease. Circulation 134, 1973-1990 (2016).

40. Le Bras, A. et al. VE-statin/egfl7 expression in endothelial cells is regulated by a distal enhancer and a proximal promoter under the direct control of Erg and GATA-2. PLoS ONE 5, e12156 (2010)

41. Wingender, E. The TRANSFAC project as an example of framework technology that supports the analysis of genomic regulation. Brief. Bioinformatics 9, 326-332 (2008).

42. Zhang, X. O., Gingeras, T. R. \& Weng, Z. Genome-wide analysis of polymerase III-transcribed Alu elements suggests cell-type-specific enhancer function. Genome Res. 29, 1402-1414 (2019).

43. Zhang, J. et al. An integrative ENCODE resource for cancer genomics. Nat. Commun. 11, 3696 (2020)

44. Harris, T. A., Yamakuchi, M., Kondo, M., Oettgen, P. \& Lowenstein, C. J. Ets1 and Ets-2 regulate the expression of microRNA-126 in endothelial cells. Arterioscler. Thromb. Vasc. Biol. 30, 1990-1997 (2010).

45. Qi, J. et al. HDAC8 inhibition specifically targets $\operatorname{Inv}(16)$ acute myeloid leukemic stem cells by restoring p53 acetylation. Cell Stem Cell 17, 597-610 (2015).

46. Zhang, Y., Woodford, N., Xia, X. \& Hamburger, A. W. Repression of E2F1mediated transcription by the ErbB3 binding protein Ebp1 involves histone deacetylases. Nucleic Acids Res. 31, 2168-2177 (2003).

47. Nguyen, D. Q. et al. The role of ErbB3 binding protein 1 in cancer: friend or foe? J. Cell Physiol. 233, 9110-9120 (2018).

48. Kim, V. N. MicroRNA precursors in motion: exportin-5 mediates their nuclear export. Trends Cell Biol. 14, 156-159 (2004).

49. de Boor, S. et al. Small GTP-binding protein Ran is regulated by posttranslational lysine acetylation. Proc. Natl Acad. Sci. USA 112, E3679-E3688 (2015). 
50. Kuo, Y. H., Gerstein, R. M. \& Castilla, L. H. Cbfbeta-SMMHC impairs differentiation of common lymphoid progenitors and reveals an essential role for RUNX in early B-cell development. Blood 111, 1543-1551 (2008).

51. Liu, L. Y. et al. Mir-126 inhibits growth of SGC-7901 cells by synergistically targeting the oncogenes PI3KR2 and Crk, and the tumor suppressor PLK2. Int J. Oncol. 45, 1257-1265 (2014).

52. Wakioka, T. et al. Spred is a Sprouty-related suppressor of Ras signalling. Nature 412, 647-651 (2001).

53. Lee, K. J. et al. Requirement for Plk2 in orchestrated ras and rap signaling, homeostatic structural plasticity, and memory. Neuron 69, 957-973 (2011).

54. Sears, R. et al. Multiple Ras-dependent phosphorylation pathways regulate Myc protein stability. Genes Dev. 14, 2501-2514 (2000).

55. Coller, H. A., Forman, J. J. \& Legesse-Miller, A. "Myc'ed messages": myc induces transcription of E2F1 while inhibiting its translation via a microRNA polycistron. PLoS Genet. 3, e146 (2007).

56. Hermeking, H. et al. Identification of CDK4 as a target of c-MYC. Proc. Natl Acad. Sci. USA 97, 2229-2234 (2000).

57. Li, Z., Boone, D. \& Hann, S. R. Nucleophosmin interacts directly with c-Myc and controls c-Myc-induced hyperproliferation and transformation. Proc. Natl Acad. Sci. USA 105, 18794-18799 (2008).

58. Fernandez, P. C. et al. Genomic targets of the human c-Myc protein. Genes Dev. 17, 1115-1129 (2003).

59. Pulikkan, J. A. et al. CBFbeta-SMMHC inhibition triggers apoptosis by disrupting MYC chromatin dynamics in acute myeloid leukemia. Cell 174, 1325 (2018).

60. $\mathrm{Li}, \mathrm{Z}$. et al. Overexpression and knockout of miR-126 both promote leukemogenesis. Blood 126, 2005-2015 (2015).

61. Mandoli, A. et al. CBFB-MYH11/RUNX1 together with a compendium of hematopoietic regulators, chromatin modifiers and basal transcription factors occupies self-renewal genes in inv(16) acute myeloid leukemia. Leukemia 28, 770-778 (2014).

62. Zhen, T. et al. RUNX1 and CBFbeta-SMMHC transactivate target genes together in abnormal myeloid progenitors for leukemia development. Blood 136, 2373-2385 (2020).

63. Nucera, S. et al. miRNA-126 orchestrates an oncogenic program in B cell precursor acute lymphoblastic leukemia. Cancer Cell 29, 905-921 (2016).

64. Castilla, L. H. et al. The fusion gene Cbfb-MYH11 blocks myeloid differentiation and predisposes mice to acute myelomonocytic leukaemia. Nat. Genet. 23, 144-146 (1999).

65. Zhao, L. et al. CBFB-MYH11 hinders early T-cell development and induces massive cell death in the thymus. Blood 109, 3432-3440 (2007).

66. Kuo, Y. H. et al. Runx2 induces acute myeloid leukemia in cooperation with Cbfbeta-SMMHC in mice. Blood 113, 3323-3332 (2009).

67. Kühn, R., Schwenk, F., Aguet, M. \& Rajewsky, K. Inducible gene targeting in mice. Science 269, 1427-1429 (1995)

68. Weng, L. et al. MicroRNA profiling of clear cell renal cell carcinoma by wholegenome small RNA deep sequencing of paired frozen and formalin-fixed, paraffin-embedded tissue specimens. J. Pathol. 222, 41-51 (2010).

69. Subramanian, A. et al. Gene set enrichment analysis: a knowledge-based approach for interpreting genome-wide expression profiles. Proc. Natl Acad. Sci. USA 102, 15545-15550 (2005).

70. Liberzon, A. in Stem Cell Transcriptional Networks (ed. Kidder, B. L.) (Springer, 2014).

\section{Acknowledgements}

This work was supported in part by the National Institutes of Health under award number R01CA205247 (to Y.-H.K. and G.M.), U01CA250046 (to Y.-H.K., G.M. and R.C.R.), R01CA213131 (to M.K.), and the Gehr Family Center for Leukemia Research. Research reported in this publication included work performed in the Analytical Cytometry Core and Animal Resource Center supported by the National Cancer Institute of the National Institutes of Health under award number P30CA33572. The content is solely the responsibility of the authors and does not necessarily represent the official views of the National Institutes of Health.

\section{Author contributions}

L.Z. and L.X.T.N. designed and performed research, analyzed data, and wrote the manuscript; Y.-C.C., D.W., G.J.C., D.H.H, C.J.B., X.H., H.D., S.L., M.L., D.Z., J.Q.,W.K.H., Q.C., E.C., W.C. and X.W. performed research, analyzed data, and reviewed manuscript; P.S. synthesized the CpG-anti-miR-126 and reviewed the manuscript; R.C.R., M.K., L.L, B.Z. and G.M. designed research and reviewed manuscript; Y.-H.K. designed research, analyzed and interpreted data, and wrote the manuscript.

\section{Competing interests}

The authors declare no competing interests.

\section{Additional information}

Supplementary information The online version contains supplementary material available at https://doi.org/10.1038/s41467-021-26420-7.

Correspondence and requests for materials should be addressed to Ya-Huei Kuo.

Peer review information Nature Communications thanks Joost Martens, Takahiro Ochiya and the other, anonymous, reviewer(s) for their contribution to the peer review of this work.

Reprints and permission information is available at http://www.nature.com/reprints

Publisher's note Springer Nature remains neutral with regard to jurisdictional claims in published maps and institutional affiliations.

pen Access This article is licensed under a Creative Commons Attribution 4.0 International License, which permits use, sharing, adaptation, distribution and reproduction in any medium or format, as long as you give appropriate credit to the original author(s) and the source, provide a link to the Creative Commons license, and indicate if changes were made. The images or other third party material in this article are included in the article's Creative Commons license, unless indicated otherwise in a credit line to the material. If material is not included in the article's Creative Commons license and your intended use is not permitted by statutory regulation or exceeds the permitted use, you will need to obtain permission directly from the copyright holder. To view a copy of this license, visit http://creativecommons.org/ licenses/by/4.0/

(C) The Author(s) 2021 